\title{
Evaluation de la mise en oeuvre et des réalisations de l'Approche 3D au sein du Plan d'Action National de Planification Familiale (PANPF) au Sénégal-Partie 2: Rapport de Synthese
}

\author{
Fatou Mbow \\ Population Council \\ Leslie Dubent \\ Population Council \\ Nafissatou Diop \\ Population Council \\ Fatou Ndiaye Turpin \\ Bocar Mamadou Daff
}

See next page for additional authors

Follow this and additional works at: https://knowledgecommons.popcouncil.org/departments_sbsr-rh

Part of the Demography, Population, and Ecology Commons, Family, Life Course, and Society Commons, International Public Health Commons, Maternal and Child Health Commons, and the Women's Health Commons How does access to this work benefit you? Let us know!

\section{Recommended Citation}

Mbow, Fatou, Leslie Dubent, Nafissatou Diop, Fatou Ndiaye Turpin, Bocar Mamadou Daff, and Babacar Mane. 2015. "Evaluation de la mise en oeuvre et des réalisations de l'Approche 3D au sein du Plan d'Action National de Planification Familiale (PANPF) au Sénégal-Partie 2: Rapport de Synthese." Dakar: Population Council, The Evidence Project. 


\section{Authors}

Fatou Mbow, Leslie Dubent, Nafissatou Diop, Fatou Ndiaye Turpin, Bocar Mamadou Daff, and Babacar Mane 


\section{Evaluation de la mise en œuvre et des} réalisations de l'Approche $3 \mathrm{D}$ au sein du Plan d'Action National de Planification Familiale (PANPF) au Sénégal

\section{PARTIE 2 : RAPPORT DE SYNTHESE}

Fatou Bintou Mbow, Chargée de Programmes, Population Council Leslie Dubent, Assistante de Recherche, Population Council Nafissatou Diop, Country Director, Population Council Fatou Ndiaye, Maternal and Child Health/Family Planning Specialist, USAID Senegal Bocar Mamadou Daff, Directeur, Direction de la Santé de la Reproduction et de la Survie de l'Enfant. Ministère de la Santé et de l'Action Sociale du Sénégal Babacar Mané, Chargé de Programmes, Population Council

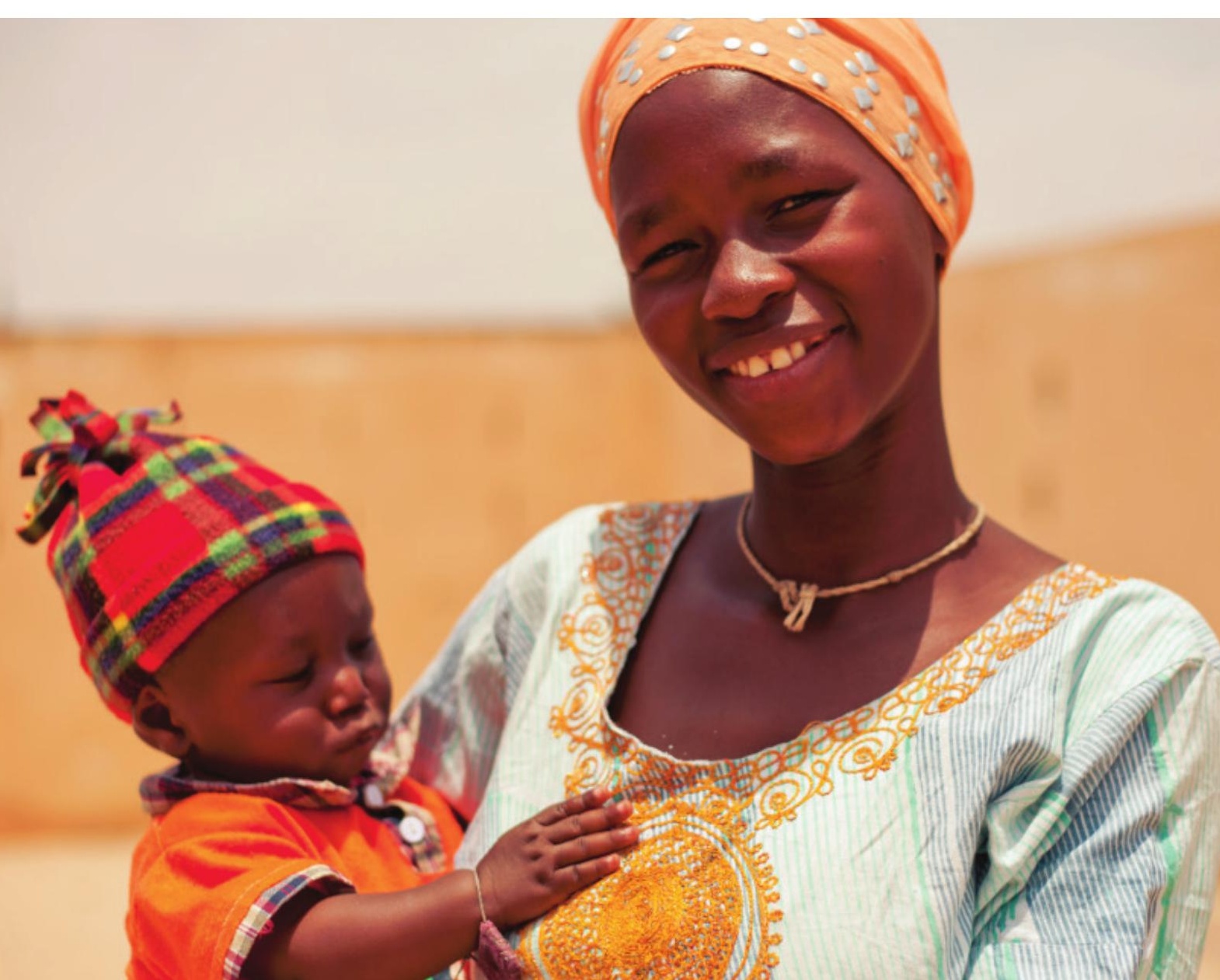




\section{The Evidence Project}

Population Council

4301 Connecticut Avenue, NW, Suite 280

Washington, DC 20008 USA

tel +12022379400

evidenceproject.popcouncil.org

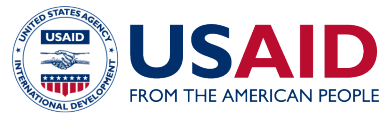

Le Projet Evidence est rendu possible grâce au généreux support du Peuple Américain à travers l'Agence des Etats Unis pour le Développement International (USAID) sous les termes de l'accord de coopération no. AID-OAA-A-13-00087. Le contenu de ce document est la seule responsabilité du Projet Evidence et ne reflète pas nécessairement les points de vue de l'USAID ou du Gouvernement des Etats Unis.

\section{Evidence}

Le Projet Evidence utilise la science de la mise en œuvre -la production, traduction, et utilisation stratégique d'évidences - pour renforcer et mettre à l'échelle des programmes de planification familiale et de santé de la reproduction afin de réduire les grossesses non désirées partout dans le monde. Le Projet Evidence est dirigé par le Population Council en partenariat avec le réseau INDEPTH, International Planned Parenthood Federation, PATH, Population Reference Bureau, et un réseau de ressources universitaires.

Publié dans Décembre 2015.

Citation recommandée: Mbow FB, Dubent L, Diop N, Ndiaye F, Daff BM \& Mané B. 2015. "Evaluation de la mise en oeuvre et des réalisations de l'Approche 3D au sein du Plan d'Action National de Planification Familiale (PANPF) au Sénégal. Partie 2: Rapport de synthèse," Rapport. Dakar, Sénégal: Population Council, Le Projet Evidence.

Crédit photo sur la page de garde (C) 2006 Richard Nyberg, Courtesy of Photoshare

(C) 2015 The Population Council, Inc. 


\section{Table des matières}

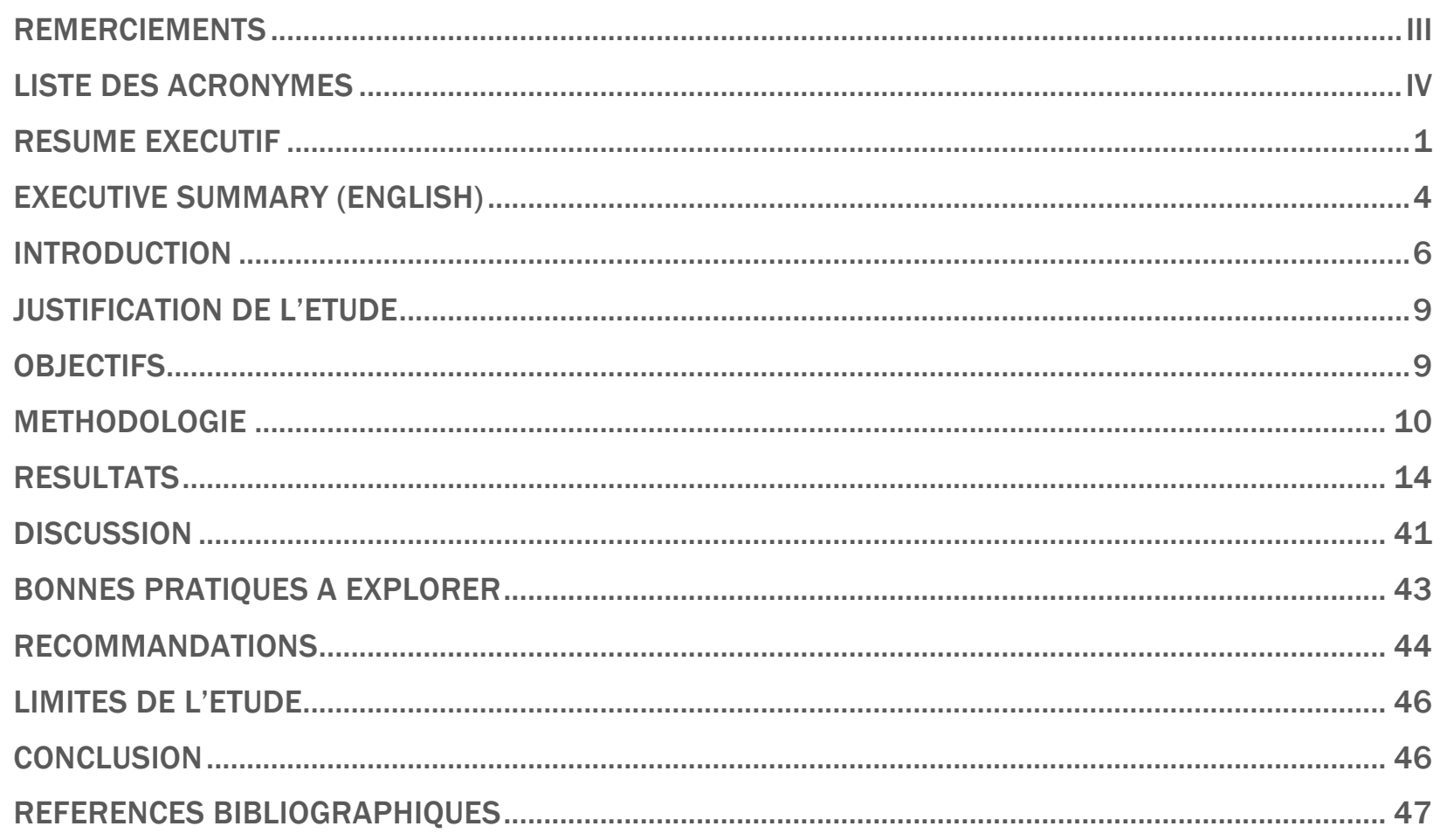




\section{Remerciements}

Les auteurs remercient toutes les institutions, organisations et personnes qui ont contribué à la réalisation de cette étude en mettant à leur disposition la documentation utile ou en les orientant vers d'autres parties prenantes susceptibles d'en détenir.

Nous remercions également toutes les parties prenantes intervenant dans le domaine de la planification familiale, aussi bien au niveau central qu'au niveau régional, pour leur précieuse collaboration à travers leur facilitation pour la mise en œuvre de l'étude et pour avoir apporté les réponses idoines à nos questions.

Nous voulons aussi témoigner notre profonde gratitude aux femmes des localités visitées qui ont accepté de participer aux groupes de discussion dirigée.

Enfin, nous saluons le professionnalisme des agents de collecte des données qui a beaucoup contribué à la réussite de cette étude. 


\section{Liste des acronymes}

$3 \mathrm{D}$

AcDev

ADC

ADEMAS

ASBEF

ASC

BNS

CLD

CRD

DIU

DMPA-IM

DMPA-SC

DPF

DSDOM

DSRSE

EA

ECD

EDS

EDS-C

EDS-MICS

GD

ICP

IEC

IPM

ISF
Démocratisation,

Démédicalisation,

Décentralisation

Action et Développement

Agent de Développement

Communautaire

Agence pour le Développement

du Marketing Social

Association Sénégalaise pour le

Bien-Etre Familial

Agent de Santé Communautaire

Besoins Non Satisfaits

Comité local de

Développement

Comité Régional de

Développement

Dispositif Intra-Utérin

Dépôt d'Acétate

Médroxyprogestérone -

Formule Intramusculaire

Dépôt d'Acétate

Médroxyprogestérone -

Formule Sous-Cutanée

Division de la Planification

Familiale

Dispensateur de Soins à

Domicile

Direction de la Santé de la

Reproduction et de la Survie de l'Enfant

Entretien Approfondi

Equipe Cadre de District

Enquête Démographique et de

Santé

Enquête Démographique et de

Santé Continue

Enquête Démographique et de

Santé à Indicateurs Multiples

Groupe de Discussion

Infirmier Chef de Poste

Information, Education et

Communication

Informed Push Model

Indice Synthétique de

Fécondité
ISSU

MCR

MLDA

MSAS

MSI

ODD

OIP

OMD

ONG

PANPF

PC

$\mathrm{PF}$

PNA

PNP

PPP

PPS

PRA

PTF

RSJ

SAFI

SPSR

SR

SRAJ

TAC

TPC

UNFPA

USAID
Initiative Sénégalaise de Santé

Urbaine

Médecin Chef de Région

Méthodes à Longue Durée

d'Action

Ministère de la Santé et de

l'Action Sociale

Marie Stopes International

Objectifs de Développement

Durable

Offre Initiale de Pilule

Objectifs du Millénaire pour le

Développement

Organisation Non-

Gouvernementale

Plan d'Action National de

Planification Familiale

Population Council

Planification Familiale

Pharmacie Nationale

d'Approvisionnement

Politiques, Normes et

Protocoles

Partenariat Public Privé

Point de Prestation de Services

Pharmacie Régionale

d'Approvisionnement

Partenaires Techniques et

Financiers

Réseau Siggil Jigeen

Sages-Femmes Itinérantes

Sécurisation des Produits de

Santé de la Reproduction

Santé de la Reproduction

Santé de la Reproduction des

Adolescents et des Jeunes

Tableaux d'Acquisition des

Contraceptifs

Taux de Prévalence

Contraceptive

Fonds des Nations Unies pour la Population

Agence des Etats Unis pour le Développement International 


\section{Résumé exécutif}

Au Sénégal, comme dans la plupart des pays d'Afrique de l'Ouest francophone, les taux de fécondité et de mortalité maternelle et infantile sont encore élevés, et le Taux de Prévalence Contraceptive (TPC) demeure faible. Au regard de ces enjeux, le Sénégal a positionné la planification familiale (PF) en tant que priorité nationale, et les autorités ont pris des engagements sur le plan du financement, de la structure organisationnelle, et de la révision des politiques de santé. Un des leaders dans le domaine de la PF en Afrique de l'Ouest francophone, le Sénégal a entrepris ces dernières années diverses initiatives afin d'atteindre ses objectifs nationaux et internationaux. Dans ce contexte, le Plan d'Action National de Planification Familiale (PANPF) 2012-2015 a été élaboré en se basant sur l'approche 3D, acronyme pour Démocratisation, Démédicalisation et Décentralisation.

Au moment où le PANPF 2012-2015 arrive à terme, et que l'élaboration d'un nouveau plan est envisagée, la documentation de l'approche 3D, de sa mise en œuvre et de ses réalisations est apparue comme une nécessité. Ainsi, en collaboration avec la Direction de la Santé de la Reproduction et de la Survie de l'Enfant (DSRSE), le Population Council a mené la présente étude. Cette étude a été réalisée dans le cadre du Projet Evidence financé par l'Agence des Etats Unis pour le Développement International (USAID).

L'étude avait pour objectifs de documenter l'approche 3D en relation avec le PANPF, décrire les leçons tirées de sa mise en œuvre et partager les bonnes pratiques avec d'autres pays du Partenariat de Ouagadougou et audelà. Il s'agissait de fournir des évidences nécessaires à la prise de décision, notamment en vue de l'élaboration du prochain plan national d'action PF.

L'étude a combiné une revue de la littérature et une recherche qualitative, à travers 26 Entretiens individuels Approfondis (EA) avec les parties prenantes de la PF et huit (8) Groupes de Discussions (GD) avec des femmes en âge de procréer, âgées de 25 à 44 ans, qui étaient utilisatrices actuelles de la PF ou l'avaient utilisée au cours des cinq (5) dernières années. Elle a été menée dans les régions de Dakar et Thiès.

\section{Résultats}

Les principaux résultats de la recherche qualitative et de la revue de la littérature se présentent pour chaque thème abordé comme suit:

- Perceptions sur la PF : la PF est globalement perçue aussi bien par les acteurs PF, que les femmes bénéficiaires comme un domaine ayant connu beaucoup d'avancées ces cinq (5) dernières années. Pour les acteurs PF cela se traduit notamment à travers l'élargissement des acteurs intervenant dans le domaine, une meilleure organisation, une meilleure coordination, une meilleure adhésion et participation des communautés aux interventions de PF (notamment hommes et leaders religieux), une meilleure accessibilité financière et géographique et un élargissement de la gamme des méthodes. Du côté des femmes, cela se manifeste par l'engouement/adhésion massive des femmes à la PF qu'elles considèrent comme un facteur de réduction des grossesses rapprochées, contribuant à leur bien-être et épanouissement et comme étant la raison principale de la baisse des décès maternels et néonataux. Il reste tout de même, pour les acteurs, quelques aspects à renforcer par rapport aux détracteurs appartenant aux communautés religieuses, aux frais additionnels au prix du service, à la faible intégration des données du secteur privé, aux nombreux cas d'abandon, à l'insuffisance du budget affecté à la PF par le Gouvernement, et à duplication d'activités par plusieurs acteurs. 
- Connaissance de l'approche 3D : les données montrent que l'approche 3D n'est connue que par environ $1 / 5$ ème des acteurs PF interviewés, et que cette méconnaissance serait liée au manque de communication autour de l'approche. Les femmes ne connaissaient pas du tout l'approche.

Connaissance des actions menées dans le cadre de chaque « $\mathbf{D} »$ : Bien que peu de personnes interrogées déclarent spontanément connaitre l'approche 3D, beaucoup parmi elles ont pu citer des actions menées dans le cadre de chaque « $\mathrm{D}$ » une fois que la définition leur en a été donnée. Ces actions sont décrites dans la revue de la littérature. Pour la Démocratisation, les principales actions reportées sont celles menées sur le plan politique, légal et règlementaire, les actions de communication, celles ayant des effets sur le coût, les actions menées pour développer le leadership et l'autonomisation des femmes, celles en faveur des jeunes, et celles relatives au Partenariat Public-Privé. Concernant la Démédicalisation, les actions reportées sont relatives à la délégation des tâches et au développement de la PF communautaire. Pour la Décentralisation, les actions reportées sont celles liées à l'offre de services, à la prise de décisions, aux formations et à l'information, à la mise en place de l'Informed Push Model (IPM) et à la stratégie des sages-femmes itinérantes (SAFI). L'implication des collectivités locales dans la construction de structures sanitaires, le financement de la PF, et le recrutement de personnels qualifiés (notamment sages-femmes) a aussi été notée. En termes de réalisations, en promouvant l'implication d'autres secteurs et acteurs, la Démocratisation permet, entre autres, d'améliorer les connaissances et perceptions sur la PF, d'harmoniser les prix des contraceptifs, d'élargir la prestation de services et d'accroitre le leadership des femmes. Grâce à la délégation des tâches et à la distribution à base communautaire, la Démédicalisation permet d'élargir les compétences des agents de santé communautaire et de rapprocher les services des populations. Finalement, en favorisant l'appropriation et la responsabilisation à tous les niveaux, la Décentralisation permet l'accès à une gamme plus large de méthodes modernes de PF, la densification des Points de Prestation des Services (PPS), l'introduction au niveau des postes de santé des Méthodes à Longue Durée d'Action (MLDA), et la disponibilité des produits jusqu'aux niveaux les plus périphériques.

- Vue globale de l'approche 3D : Au-delà des divergences d'opinions en ce qui concerne le «D » qui aurait le plus progressé, les acteurs PF et les femmes bénéficiaires s'accordent à dire que les 3D sont complémentaires et interdépendants. L’approche est perçue comme bonne du fait, entre autres, de la dimension multisectorielle qu'elle intègre. Il s'agit pour eux d'une approche innovante qui gagnerait à être systématisée et mieux vulgarisée pour une bonne appropriation par les parties prenantes. Les acteurs PF sont tellement séduits par cette approche et ses résultats qu'ils pensent à l'unanimité qu'elle devrait être reconduite pour servir de ligne directrice à l'élaboration du prochain PANPF.

- Effets perçus de l'approche 3D : Parmi les principaux effets que les personnes interrogées attribuent à la mise en œuvre de l'approche $3 \mathrm{D}$, on peut noter : une meilleure accessibilité géographique et financière aux services PF, la hausse du TPC, le renforcement des capacités des acteurs communautaires, une meilleure acceptation/adhésion à la PF, la disponibilité des produits, l'amélioration des connaissances sur la PF, l'espacement des naissances et la baisse de la mortalité maternelle et infantile.

- Pérennisation de l'approche 3D : l'étude montre que la pérennisation de l'approche 3D nécessite une bonne sensibilisation sur l'approche, une meilleure implication et appropriation par les parties prenantes, la mise en place de stratégies pour la sécurisation des produits, et la motivation des agents de santé communautaire ainsi que leur formation continue.

Recommandations : Des recommandations ont été formulées à l'endroit de l'Etat/du Ministère de la Santé et de l'Action Sociale (MSAS), des acteurs PF et partenaires techniques, et des partenaires financiers. 
Conclusion : L'approche 3D est peu connue en tant que concept bien que les parties prenantes l'opérationnalisent dans leurs activités de tous les jours. Les réalisations liées aux $3 \mathrm{D}$ ont contribué à la nette amélioration des indicateurs et l'approche présente des promesses fortes pour l'atteinte des objectifs de développement du Sénégal. Sa clarification et prise en compte dès l'élaboration du prochain PANPF s'avèrent indispensables pour booster encore plus rapidement la PF pour améliorer la santé des populations et réduire la mortalité maternelle, néonatale et infantile. 


\section{Executive Summary (English)}

In Senegal, as in most Francophone West African countries, rates of fertility and of maternal and infant mortality are still high, and the Contraceptive Prevalence Rate (CPR) remains low. In light of these challenges, Senegal has positioned family planning (FP) as a national priority, and authorities made commitments related to financing, organizational structure, and review of health policies related to FP. One of the leaders in FP in Francophone West Africa, Senegal has undertaken in recent years various initiatives to reach its national and international goals. In this context, the 2012-2015 National Family Planning Action Plan (NFPAP) was developed based on the 3D approach, which stands for Democratization, De-medicalization and Decentralization.

As the 2012-2015 NFPAP is coming to an end and the development of a new plan is envisioned, it is critical to document the 3D approach, its implementation, and its achievements. Thus, in collaboration with the Directorate of Reproductive Health and Child Survival (DRHCS), the Population Council conducted this study under the Evidence Project, with funding from the United States Agency for International Development (USAID).

This study aims to document the 3D approach in relation to the NFPAP, to describe lessons learned from its implementation, and to share good practices with other countries, particularly those in the Ouagadougou Partnership. This study is intended to provide evidence to support decision-making, especially for the elaboration of the next FP national action plan.

This study combines a literature review and qualitative research, including 26 in-depth interviews (IDI) with FP stakeholders and eight focus-group discussions (FGDs) with women between the ages of 25 and 44 who were currently using FP or had used FP during the past five years. This study was conducted in the Dakar and Thiès regions.

\section{Results}

The main findings from the qualitative research and the literature review are presented for the following key topics:

- Perceptions about FP: FP is generally perceived by both FP stakeholders and female FP users as an area that has advanced considerably over the past five years. For FP stakeholders, this is particularly visible through an increase in FP stakeholders, better organization, better coordination, better community support for and participation in FP interventions (especially men and religious leaders), better financial and geographical accessibility, and a broader methods mix. Female FP users cited the keen interest and strong support of women for FP, and the perception that FP has helped reduce closely spaced pregnancies, contributed to women's well-being and improved health, and is the main reason for the decrease in maternal and neonatal deaths. FP stakeholders feel that some issues remain to be addressed, including criticism from religious communities, costs added to service charges, weak integration of data from the private sector, high rates of discontinuation, lack of budget allocated to FP by the government, and duplication of activities by multiple actors.

- Knowledge of the 3D approach: The findings show that only one-fifth of the FP stakeholders know of the 3D approach, and that this lack of knowledge is linked to the lack of communication around the approach. Women did not know about the approach at all. 
Knowledge of actions conducted within each "D": Although few respondents knew the 3D approach, many were able to list actions conducted as part of each "D," once they were given the definition. The definitions of each "D" are described in the literature review. For Democratization, the key actions are those undertaken on political, legal and regulatory grounds, actions for communication, those that have an effect on cost, actions for women's leadership and empowerment, those in support of youth, and those related to public-private partnership. For De-medicalization, actions include those related to task shifting and development of FP at the community level. For Decentralization, actions reported are those related to service provision, decision-making, training and information, the implementation of the Informed Push Model (IPM), and the Itinerant Midwives Strategy (IMS). The involvement of local authorities in building health facilities, funding FP, and recruiting qualified personnel (including midwives) was also mentioned. As for achievements, by fostering the involvement of other sectors and actors, Democratization allows, among other things, improvement of knowledge and perceptions about FP, harmonization of the price of contraceptives, expansion of service delivery, and increase of women's leadership. Through task shifting and community-based distribution, Demedicalization helps broaden the skills of community health workers and bring services closer to the population. Lastly, by fostering ownership and accountability at all levels, Decentralization allows access to a broader range of modern FP methods, multiplication of Service Delivery Points (SDP), introduction of long-acting reversible contraceptives (LARCs) at the health post level, and improved product availability at the most remote levels.

- Overview of the 3D approach: Beyond some differences in opinion about which "D" might have contributed most to the progress made, FP stakeholders and female FP users agree that the three Ds are complementary and interdependent. The $3 \mathrm{D}$ approach is perceived as a good approach because, among other things, of the multi-sectoral dimension it integrates. Respondents see it as an innovative approach that should be systematized and better disseminated for broader stakeholder ownership. FP stakeholders were impressed by this approach and its results, and feel that it should be extended to serve as guidance for developing the next NFPAP.

- Perceived effects of the 3D approach: Among the main effects that respondents attribute to the implementation of the 3D approach are: better geographical and financial access to FP services, increase in CPR, capacity building of community-level actors, better acceptance of and support for FP, availability of products, improved knowledge about FP, improved birth spacing, and reduction of maternal and infant mortality.

- Sustainability of the 3D approach: the study shows that sustainability of the 3D approach requires good awareness of the approach, better involvement and adoption by stakeholders, implementation of strategies for FP commodities security, and motivation and continued training of community health workers.

Recommendations: Recommendations are made to the State/Ministry of Health and Social Action (MHSA), to FP stakeholders and technical partners, and to donors.

Conclusion: The 3D approach is a little-known concept, although FP stakeholders operationalize it in their daily activities. Achievements linked to the three Ds have contributed to a clear improvement of indicators, and the approach presents great promise for achieving Senegal's development goals. Its clarification and inclusion as part of the development of the next NFPAP are essential to more quickly improve the status of FP and, therefore, to improve the health of the population and to reduce maternal, newborn and child mortality. 


\section{Introduction}

Au Sénégal, comme dans la plupart des pays d'Afrique de l'Ouest francophone, les taux de fécondité et de mortalité maternelle et infantile sont encore élevés, et le Taux de Prévalence Contraceptive (TPC) demeure faible. La planification familiale (PF), en permettant d'éviter les grossesses non désirées ou trop rapprochées et les avortements à risque, est reconnue comme une stratégie efficace pour améliorer la santé de la mère et de l'enfant tout en contribuant au développement économique et social du pays.

C'est pourquoi, conscient de ces enjeux, le Sénégal a positionné la PF en tant que priorité nationale. Les autorités ont pris des engagements sur les plans financier et organisationnel, ainsi que sur l'ajustement des politiques de santé. L'adoption du Plan d'Action National de Planification Familiale (PANPF) 2012-2015 est une preuve de cet engagement. Les objectifs ambitieux de ce PANPF sont orientés vers l'atteinte des Objectifs du Millénaire pour le Développement (OMD) et plus récemment des Objectifs de Développement Durable (ODD). L'approche 3D, qui repose sur les concepts de Démocratisation, Démédicalisation et Décentralisation de la PF, constitue le socle de ce PANPF.

\section{HISTORIQUE DES PROGRAMMES DE PF AU SENEGAL}

Bien qu'une loi française de 1920 ait interdit la promotion des contraceptifs au Sénégal jusqu'en 1980, les premiers services de PF ont été introduits à Dakar en 1964 à la Clinique privée de la Croix Bleue, puis dans la première clinique de l'Association Sénégalaise pour le Bien-Être Familial (ASBEF) à partir de 1975. Dès l'abrogation de cette loi, ce qui constitue une manifestation de la volonté des pouvoirs publics en faveur de la PF, plusieurs programmes nationaux ont été mis en œuvre, à savoir : le "Projet Population et Santé de la Famille », financé par l'Agence des Etats Unis pour le Développement International (USAID), et le « Projet de bien-être familial » financé par le Fonds des Nations Unies pour la Population (UNFPA). En 1988, la Déclaration de Politique de la Population, qui prône l'espacement des naissances et non leur limitation, était la première politique démographique de la sous-région, à l'origine des progrès futurs en matière de PF. En 1991, le Programme National de PF est lancé. En 2009, le Plan National de Développement Sanitaire (PNDS) qui préconise la PF comme l'une des stratégies les plus efficientes pour sauver des vies et garantir l'amélioration de la santé de la mère, du nouveau-né, de l'enfant et de l'adolescent est adopté. Par ailleurs, la feuille de route multisectorielle de lutte contre la mortalité maternelle et néonatale inscrit la PF comme une des stratégies majeures. L'adoption de la loi de 2005 sur la santé de la reproduction (SR) a, en plus des documents d'orientation technique, constitué une décision importante qui a contribué à la création d'un environnement politique, juridique et réglementaire plus favorable pour la mise en œuvre du programme PF. Cette loi, précise de manière claire, les droits spécifiques accordés aux citoyens sénégalais en matière de SR/PF.

Sur le plan institutionnel, le Service National de la Santé de la Reproduction est créé en 1997. Cette structure chargée des questions liées à la SR et de l'élaboration de documents cadres donnera naissance à la Division de la Santé de la Reproduction (DSR) en 2001. La Division est devenue la Direction de la Santé de la Reproduction et de la Survie de l'Enfant (DSRSE) en 2012, donnant ainsi une nouvelle perspective pour une intégration effective des interventions en matière de santé maternelle, de PF et de survie de l'enfant.

\section{ENGAGEMENT POLITIQUE DU SENEGAL POUR LA PF}

La succession des différents programmes et l'évolution sur le plan légal et réglementaire de la SR marquent les efforts constants des pouvoirs publics pour créer un environnement politique, juridique et social plus favorable pour le développement de la PF. Depuis les années 2011-2012, ces efforts se sont consolidés et les initiatives pour le repositionnement de la PF se sont multipliées. 
Le Sénégal a adhéré aux engagements internationaux récents sur la PF. Ainsi, en juillet 2012, lors du Sommet de Londres sur la PF, Madame la Ministre de la Santé et de l'Action Sociale a réaffirmé la volonté du Gouvernement sénégalais de faire de la PF une priorité nationale. Ainsi, le Sénégal s'est fixé des objectifs ambitieux en termes d'augmentation du TPC et de diminution des Besoins Non Satisfaits (BNS). Il s'est également engagé à accroître l'allocation budgétaire annuelle à la SR de 2,5 à 5 pour cent, à augmenter de 200 pour cent l'allocation budgétaire pour les produits contraceptifs (de 100 à 300 millions de FCFA), et à augmenter le budget pour la gestion du programme de PF de 100 pour cent. L'Etat a commencé à concrétiser ses promesses en augmentant de 100 millions de FCFA par an les ressources financières pour la sécurisation des produits SR/PF et en créant une ligne budgétaire spécifique à la SR/PF de 228 millions de FCFA.

En 2012, s'inscrivant dans cette volonté de repositionnement de la PF, le Sénégal a érigé la Division de la Santé de la Reproduction (DSR) en Direction de la Santé de la Reproduction et de la Survie de l'Enfant (DSRSE) ayant en son sein la Division de la Planification Familiale (DPF). Cette Division coordonne la mise en œuvre du PANPF, en s'appuyant sur le Comité Technique PF qui regroupe les partenaires intervenant dans la mise en œuvre de ce plan. Les comités thématiques «Création de la demande ", "Offre de services ", "Sécurisation des produits contraceptifs » et « Monitoring de la performance du PANPF (gestion des données) » apportent une assistance dans leur domaine de compétence.

Dans le cadre des initiatives visant la promotion de la PF au Sénégal, la DPF a initiée la Journée Nationale de la Planification Familiale avec le soutien de l'UNFPA, IntraHealth et du projet Initiative Sénégalaise de Santé Urbaine (ISSU). Ces journées constituent des moments forts de mobilisation des acteurs et bénéficiaires autour de la PF. Les Journées Nationales se sont tenues régulièrement entre 2008 et 2013, mais depuis lors, aucune manifestation n'a eu lieu dans le cadre de cette initiative.

\section{EVOLUTION DES INDICATEURS PF}

Depuis 2005, l'Indice Synthétique de Fécondité (ISF) a tendance à se stabiliser autour de 5,0 enfants par femme. Les taux de mortalité maternelle et infanto-juvénile ont, en revanche, connu une diminution progressive, passant respectivement de 401 à 392/100 000 naissances vivantes et de 121 à 54/1 000 entre l'Enquête Démographique et de Santé à Indicateurs Multiples (EDS-MICS) de 2005 et l'EDS continue (EDS-C) de 2014.

Le TPC est généralement un bon indicateur de l'efficacité des programmes de PF. Aussi, après avoir augmenté graduellement jusqu'en 2010 (de 8 pour cent en 1997 à 10 pour cent en 2005, puis à 12 pour cent en 2010), il a fait des bonds annuels de quatre (4) points de pourcentage pendant la période de mise en œuvre du PANPF 2012-2015, pour atteindre 16 pour cent en 2012-2013 et 20 pour cent en 2014.

Cependant, les BNS en PF demeurent encore importants parmi les femmes en union. En effet, selon l'EDS-C 2014, 25,6 pour cent des femmes présentent un BNS, dont 19,2 pour cent qui souhaitent espacer leur prochaine naissance d'au moins deux (2) ans et 6,3 pour cent qui ne désirent plus de grossesse du tout, sans toutefois utiliser une méthode contraceptive.

Chez les adolescent(e)s/jeunes, qui représentent une cible importante pour les programmes de PF, le TPC pour les méthodes modernes reste encore faible même si l'utilisation de la PF par cette catégorie de la population a connu des progrès significatifs: pour les 15-19 ans il est passé de 1,9 pour cent en 2010-2011 à 12,3 pour cent en 2014, et pour les $20-24$ ans il est passé de 6,0 pour cent à 12,2 pour cent pendant la même période.

\section{NAISSANCE ET DEFINITION DE L'APPROCHE 3D}

Le Sénégal a présenté l'approche 3D pour la première fois dans la sous-région à la Conférence « Population, Développement et Planification Familiale en Afrique de l'Ouest : L’Urgence d'Agir » tenue à Ouagadougou en 
février 2011. Cette conférence réunissait les représentants de huit (8) pays de l'Afrique de l'Ouest francophone ${ }^{1}$ (Bénin, Burkina Faso, Guinée, Mali, Mauritanie, Niger, Sénégal et Togo) et leurs partenaires techniques et financiers. Dans le cadre d'un accord sur la nécessité de la prise de mesures concrètes pour accélérer l'utilisation des services PF dans ces pays, cette conférence a donné jour au Partenariat de Ouagadougou (PO).

Le Sénégal a présenté l'approche 3D, acronyme pour Démocratisation, Démédicalisation et Décentralisation. Pour la PF, à travers l'approche 3D :

- La « démocratisation » est conçue pour faciliter l'accès aux services par le biais d'une approche multisectorielle et participative;

- La « démédicalisation » vise à permettre à des agents non médicaux de fournir une gamme variée de services PF aux populations vulnérables (rurales, urbaines défavorisées, pauvres et jeunes), notamment par le biais d'une distribution à base communautaire;

- La « décentralisation » vise à consolider les systèmes de santé aux niveaux régional, district et communautaire, tout en renforçant la bonne gouvernance, la responsabilisation et la collaboration intersectorielle.

\section{PLAN D'ACTION NATIONAL DE PF (PANPF) 2012-2015}

Suite à la Conférence de Ouagadougou, le Gouvernement du Sénégal a élaboré avec ses partenaires le PANPF 2012-2015 qui a été lancé le 28 novembre 2012. Le Sénégal était alors l’un des premiers pays du PO à mettre en œuvre un tel plan afin d'offrir à toutes les femmes une équité dans l'accès à des services de PF de qualité. Son objectif ambitieux vise à augmenter le TPC pour les femmes en union des 12 pour cent reportés par l'EDSMICS 2010-2011 à 27 pour cent en 2015. Dans cette optique, 350000 nouvelles utilisatrices de méthodes contraceptives modernes devaient être recrutées : 125000 par le secteur public, 100000 par le secteur privé, et 125000 par le niveau communautaire.

L'approche stratégique principale du plan repose sur la mise en œuvre de l'approche 3D et la mise en œuvre au niveau opérationnel est basée sur six (6) domaines prioritaires pour permettre :

- La disponibilité des produits contraceptifs par la mise à l'échelle de stratégies innovantes, la distribution à base communautaire des méthodes à courte durée d'action avec un renforcement de la délégation des tâches, l'élargissement de l'offre avec l'offre initiale de pilules (OIP) et d'injectables (OII) afin de toucher au mieux les populations les plus défavorisées ;

- L'implication des acteurs du secteur privé dans l'élargissement de l'offre et la promotion de l'utilisation des contraceptifs, notamment à travers le marketing social, la mise en place d'un réseau de franchises sociales et de cliniques mobiles ;

- L'amélioration de l'offre, en particulier pour les Méthodes à Longue Durée d'Action (MLDA) ;

- Dans le système public, un service facilement accessible, de qualité et assurant la discrétion et une prise en charge appropriée des femmes et particulièrement des jeunes.

La Côte d'Ivoire a rejoint l'initiative plus tard. 


\section{Justification de l'étude}

Le PANPF 2012-2015 arrivant à terme, il existe un besoin de documenter la mise en œuvre et les réalisations de l'approche 3D qui le sous-tend. La présente étude s'inscrit dans cette dynamique. En procédant à la documentation de l'approche 3D par le biais d'une revue documentaire et d'une recherche qualitative, l'étude vise à fournir les évidences nécessaires à la prise de décision, notamment en vue de l'élaboration du prochain plan national d'action PF.

\section{Objectifs}

Cette étude vise à documenter l'approche 3D en relation avec le PANPF, à décrire les leçons tirées de la mise en œuvre de cette approche au sein du PANPF et à partager les bonnes pratiques avec d'autres pays du Partenariat de Ouagadougou et au-delà. De manière spécifique :

La revue documentaire a eu pour objectifs de :

- Evaluer et analyser la documentation existante et disponible ;

- Identifier les institutions et personnes jouant un rôle clé dans les réalisations du plan afin d'approfondir l'analyse de la mise en œuvre de l'approche à travers des interviews (recherche qualitative) ;

- Focaliser la documentation sur les aspects ayant jusqu’à présent reçu le moins d’attention.

La recherche qualitative, quant à elle, a eu pour objectifs de compléter les données recueillies lors de la revue de la littérature, en :

- Documentant les contributions principales de l'approche 3D à la PF dans le cadre du PANPF du point de vue des acteurs PF et des femmes bénéficiaires interrogés ;

- Etudiant comment les résultats de la mise en œuvre de l’approche ont pu contribuer à l'amélioration des indicateurs PF;

- Explorant comment le transfert de tâches et les activités d'offre de services à base communautaire, qui ont été sélectionnés comme étant des interventions à haut impact et mises en œuvre dans le cadre des stratégies de la «Démédicalisation » et de la «Décentralisation » dans le programme PF et inclus dans le PANPF, ont pu améliorer l'offre des services PF, avec une concentration particulière sur l'équité.

Bien que faisant la synthèse de toute l'étude, y compris la revue documentaire, le présent rapport met un accent particulier sur la composante recherche qualitative, la revue de la littérature ayant fait l'objet d'un rapport distinct intitulé «Evaluation de la mise en œuvre et des réalisations de l'Approche 3D au sein du Plan d'Action National de Planification Familiale (PANPF) au Sénégal - Revue documentaire ». 


\section{Méthodologie}

\section{REVUE DOCUMENTAIRE}

La revue documentaire a consisté en un inventaire de la documentation existante auprès des parties prenantes et une recherche en ligne. Une lecture et une analyse approfondies des notes et documents obtenus ont ensuite permis d'extraire les éléments d'information sur la mise en œuvre de l'approche 3D. Le rapport de la revue présente la méthodologie en détails.

\section{RECHERCHE QUALITATIVE}

\section{Conception de l'étude}

Deux (2) régions ont été choisies comme sites de l'étude, à savoir Dakar et Thiès. Pour la région de Thiès, il s'agit plus précisément des districts sanitaires de Thiès, Mbour, Thiadiaye et Popenguine. Le choix de ces deux (2) régions a été motivé par le fait que :

- Dakar, capitale du pays, regroupe la plupart des institutions et organisations nationales et internationales intervenant dans le programme PF.

- La région de Thiès a été choisie du fait de l’importance de son réseau communautaire et des activités qui y ont été déployées en matière de PF. De plus, cette région présente, après Dakar, le plus important taux d'utilisation de contraception moderne ( 24,8 pour cent). Le taux de demande satisfaite par les méthodes modernes est aussi le deuxième plus important du pays, atteignant 48,4 pour cent (ANSD et ICF International, 2015).

\section{Population de l'étude}

Les populations de l'étude sont:

- Les acteurs PF : il s'agit notamment de :

- Décideurs politiques, en particulier les parlementaires ;

- Représentants du Gouvernement,

- Autorités locales impliquées dans chacun des « D», notamment les membres des équipes cadre de régions et de districts ;

- Responsables et gestionnaires de programmes/projets ;

- Représentants d'organisations non-gouvernementales (ONG) nationales et internationales; et

- Représentants d'organisations de la société civile (OSC).

- Les femmes en âge de procréer, âgées de 25 à 44 ans, utilisatrices actuelles de la PF ou l'ayant utilisée au cours des cinq (5) dernières années. Elles sont aussi appelées femmes bénéficiaires dans ce rapport. 


\section{Procédures de collecte des données et de recrutement}

\section{Outils de collecte}

Trois (3) outils de collecte ont été développés, à savoir :

- Guide d'Entretiens Approfondis (EA) avec les acteurs PF. Ce guide est basé sur des informations générales sur le type d'acteur, sa perception sur la PF, sa connaissance de l'approche 3D, les effets perçus de l'approche 3D, sa perception sur la durabilité de l'approche, et sa vue globale de l'approche 3D.

- Guide de Groupes de Discussions (GD) avec les femmes bénéficiaires. Ce guide est basé sur des questions relatives à leurs perceptions sur les changements dans le domaine de la $\mathrm{PF}$ au cours des cinq (5) dernières années, leur connaissance de l'approche 3D, les effets perçus de l'approche 3D, leurs perceptions sur la durabilité du progrès, et les leçons à retenir pour la programmation PF.

- Formulaire de collecte de données sur les caractéristiques des participantes aux GD et leur expérience par rapport à l'utilisation de la PF.

\section{Recrutement des participants et déroulement de la collecte}

\section{Entretiens Approfondis}

Dans la région de Dakar, les EA ont été menés par l'équipe de recherche du Population Council et se sont déroulés entre le 18 août et le 9 septembre 2015. L’identification des acteurs clés a été initialement effectuée en collaboration avec les partenaires de l'étude, en l'occurrence la DSRSE et l'équipe du Bureau Santé de l'USAID Sénégal. Les acteurs identifiés ont été contactés par courrier officiel pour solliciter le recueil de documents disponibles à leur niveau. Ils ont ensuite été relancés pour une demande de participation à un entretien approfondi. Un suivi a été effectué par contact téléphonique pour les acteurs ne répondant pas à la demande écrite. D’autres acteurs ont aussi été identifiés par effet boule de neige. La sélection a cessé lorsque tous les acteurs contactés ont été interrogés, ont fait part de leur indisponibilité ou n'ont plus répondu aux sollicitations écrites ou téléphoniques à une date arrêtée par l'équipe de recherche.

Dans la région de Thiès, les EA se sont déroulés du 30 septembre au 13 octobre 2015 auprès des responsables de la Région Médicale, des districts sanitaires de Thiès, Mbour, Thiadiaye et Popenguine, et d'autres acteurs PF. Au début du processus, une lettre d'information a été envoyée par la DSRSE à la Région Médicale. Ensuite, une lettre officielle a été envoyée à chaque acteur PF identifié pour expliquer l'étude et solliciter un rendez-vous pour un entretien.

Au début de chaque entretien, un formulaire de consentement éclairé a été lu et signé par le participant de manière volontaire. Les entretiens ont duré en moyenne une (1) heure et ont été enregistrés avec leur permission. Tous les entretiens ont été menés en face à face dans un endroit choisi par le répondant.

\section{Groupes de discussions dirigées}

Les GD ont été organisés dans les districts sanitaires de Mbour, Thiadiaye et Popenguine du 5 au 16 octobre 2015. Ces GD ont été menés par quatre (4) agents de collecte recrutés à cet effet. Afin de faciliter les discussions sur le sujet sensible de la PF, les agents de collecte étaient toutes des femmes. Elles ont reçu une formation portant d'abord sur la présentation de l'étude 3D et les aspects relatifs à la conduite d'une recherche qualitative et à l'éthique. La formation a été aussi l'occasion de se familiariser avec les outils de collecte, de procéder à leur traduction en wolof, et de prétester tous les outils avant la collecte effective. Les agents de collecte ont ensuite été réparties en deux (2) équipes, composées chacune d'une modératrice et d'une observatrice. 
Les critères de sélection des participantes aux GD étaient: i) avoir entre 25 et 44 ans ; ii) utiliser actuellement la PF ou l'avoir utilisé à un moment donné au cours des cinq (5) dernières années ; iii) avoir obtenu sa méthode contraceptive dans un établissement public, ou auprès d'un agent de santé communautaire, d'une ONG ou d'une structure privée à but non lucratif ; iv) accepter que les autres participantes au GD apprennent son statut PF.

Pour le recrutement des participantes, les coordonnatrices SR ont facilité le contact avec des acteurs communautaires (relais, matrones, bajenu gox, Agents de Santé Communautaire (ASC)) afin de solliciter leur appui pour l'identification de femmes éligibles. Après constitution d'une liste des candidates potentielles identifiées par les acteurs communautaires, les femmes ont été sélectionnées par échantillonnage aléatoire simple. Etant donné que la pratique de la PF est souvent dissimulée au Sénégal, des rencontres dans les structures de santé ont été préférées aux visites à domicile pour la vérification des critères d'éligibilité et l'explication de l'étude par l'équipe de recherche. Si la femme acceptait de participer, des informations lui étaient données sur la date et l'heure du GD. Si elle ne voulait ou ne pouvait participer, elle était remerciée, et le processus se répétait jusqu’à ce qu’au moins huit (8) femmes aient accepté de participer au GD.

Les GD ont été menés en wolof et ont été enregistrés après accord explicite des participantes. Les données personnelles de chaque participante ont été recueillies individuellement. Chaque GD a débuté par la lecture, l'explication et la signature du formulaire de consentement éclairé. Ensuite, la modératrice a expliqué à nouveau l'étude et les règles de fonctionnement du GD. Durant la discussion proprement dite, la modératrice a été chargée d'animer la séance et l'observatrice de prendre des notes et d'enregistrer la discussion. Les séances de discussion ont duré en moyenne 1 h 30.

\section{Difficultés rencontrées lors de la collecte}

Lors de la collecte des données, les équipes ont rencontré quelques difficultés sur le terrain, notamment :

- Conflit d'agenda de la collecte avec celui des acteurs locaux, ces derniers étant souvent contraints par leurs propres activités (réunions, revues, ateliers de formation...).

- Difficulté pour les acteurs communautaires de respecter les critères d'éligibilité en établissant la liste des femmes proposées pour les GD (notamment l'âge et l'estimation de la période d'utilisation de la $\mathrm{PF})$, ce qui a parfois causé des retards dans le recrutement des participantes.

\section{Gestion des données}

Une attention particulière a été portée à la gestion des données. Les EA et GD ont été enregistrés puis transcrites en français sur Microsoft Word. Pour préserver l'anonymat des participants et la confidentialité de toute information qui permettrait d'identifier les répondants et de les localiser, un système de cryptage a été établi. Par ailleurs, les transcriptions anonymisées ainsi que les fichiers audio des EA et GD ont été conservés dans un endroit sécurisé conformément aux critères de protection des données du Population Council. Seule l'équipe de recherche peut accéder aux données.

\section{Analyse des données}

L'analyse de contenu a été utilisée pour l'interprétation des données. Elle a combiné une démarche descriptive et une démarche inductive, en vue de présenter un état des lieux d'une part et d'autre part, de déterminer les thèmes ainsi que les modèles qui ont permis de construire des typologies. Cela a permis de répondre de manière plus fine aux besoins d'analyse qui tiennent compte des différentes orientations définies dans le protocole de recherche.

Les données quantitatives collectées, notamment sur les caractéristiques sociodémographiques des participantes aux GD et leur expérience par rapport à l'utilisation de la PF, ont été analysées sur Epi info 7. 


\section{Aspects éthiques}

\section{Approbation éthique}

Le protocole de recherche a reçu l'approbation éthique du Comité d'Ethique du Population Council et celle du Comité National d'Ethique pour la Recherche en Santé (CNERS) du MSAS, ainsi qu'une Autorisation Administrative de la Direction de la Planification, de la Recherche et des Statistiques du MSAS.

\section{Consentement éclairé}

Le consentement écrit, libre et éclairé, a été obtenu de chaque répondant aux EA et de chaque participante aux GD. Les informations et exigences liées à la participation à l'étude ont été lues aux participants qui ont exprimé leur accord et signé le formulaire de consentement éclairé.

\section{Confidentialité}

Plusieurs mesures ont été prises afin de garantir la confidentialité des EA et des GD: ils ont été réalisés par des intervieweurs expérimentés, dans des lieux le plus privé possible; le lieu précis n’est noté dans aucune publication ; les ordinateurs sont protégés par des mots de passe ; les notes et les enregistrements sont gardés dans des placards verrouillés au bureau du Population Council. Une fois la transcription complétée et vérifiée et le rapport d'étude publié, les notes et les enregistrements seront détruits.

\section{Anonymat}

Pour s'assurer de l'anonymat des répondants et des informations collectées, aucune identification n'a été enregistrée sur les notes d'entretien, les enregistrements ou les transcriptions électroniques. Les données sont gardées séparément des identifiants. L'anonymat des participantes lors des GD a été assuré grâce à l'utilisation de numéros pour leur identification. 


\section{Résultats}

\section{BILAN DE LA COLLECTE DES DONNEES}

Dans le cadre de la revue documentaire, huit (8) sites web ont été consultés. Six (6) présentations et 33 documents ont été recueillis, parmi lesquels des publications, des rapports (d'études, d'activités), des articles, des bulletins d'information, et des plans/documents stratégiques.

Concernant la recherche qualitative, 26 entretiens approfondis ont été réalisés, dont 15 à Dakar et 11 à Thiès. Le tableau ci-dessous en dresse le bilan.

\section{TABLE 1 | BILAN DAS ENTRETIENS APPROFONDIS}

\begin{tabular}{|l|l|c|}
\hline \multirow{1}{*}{ Site } & \multicolumn{1}{|c|}{ Population Cible } & Nombre \\
\hline \multirow{4}{*}{ Dakar } & Parlementaire & 1 \\
\cline { 2 - 3 } & Autorités sanitaires niveau central (Représentants du gouvernement) & 4 \\
\cline { 2 - 3 } & Partenaires techniques et financiers (PTF) & 2 \\
\cline { 2 - 3 } & Représentants d'ONG & 7 \\
\cline { 2 - 3 } & Membre de réseau d'ONG & 1 \\
\hline \multirow{4}{*}{ Thiès } & Décideur politique & 5 \\
\cline { 2 - 3 } & Autorités sanitaires locales & 1 \\
\cline { 2 - 3 } & PTF & 4 \\
\cline { 2 - 3 } & Membres ECD & $\mathbf{2 6}$ \\
\hline \multirow{2}{*}{ Total } & & 1 \\
\hline
\end{tabular}

En ce qui concerne les GD, un total de 80 femmes ont été atteintes à travers huit (8) GD réalisés avec des groupes de 8 à 12 femmes. Les femmes ont été divisées selon deux (2) groupes d'âges: 25-34 ans et 35-44 ans.

Les données disponibles caractéristiques sur des participantes indiquent que la plupart des femmes étaient mariées (75), et les autres (3) divorcées. Elles étaient toutes de religion musulmane. Le nombre d'enfants vivants était en moyenne de 3,9 (entre 0 et 9 enfants). 16 femmes avaient atteint un niveau d'étude secondaire, 28 un niveau primaire et 34 n'avaient aucun niveau d'étude ou étaient issues de l'école coranique. 34 femmes étaient vendeuses ou commerçantes, 30 étaient des femmes au foyer. Il y avait aussi des couturières, relais, agents de santé, infirmière, restauratrice et élève.

Par rapport à leur expérience de la PF, 69 femmes étaient des utilisatrices actuelles et neuf (9) l'avaient utilisé au cours des cinq (5) dernières années. Parmi les utilisatrices actuelles, 35 avaient opté pour l'injectable, 17 pour l'implant, 14 pour la pilule, une (1) pour le Dispositif Intra-Utérin (DIU) et une (1) avait eu recours à la stérilisation féminine. Les femmes utilisaient leur méthode depuis quatre (4) ans en moyenne. 29 femmes n'utilisaient aucune méthode avant de recourir à leur méthode actuelle. La majorité avait obtenu leur méthode auprès d'une sage-femme (64) ou dans un établissement de santé public (67). Deux (2) avaient bénéficié des services d'une clinique mobile. 
Parmi les anciennes utilisatrices, cinq (5) avaient eu recours à l'injectable, trois (3) à la pilule et une (1) à l'implant. Sept (7) femmes avaient arrêté la PF pour un désir de grossesse, les deux (2) autres par crainte d'effets potentiels liés à une longue utilisation ou devant une absence de règles.

Deux (2) GD ont été menés avec chaque groupe d'âge en milieu urbain/périurbain et en milieu rural. Le tableau ci-dessous fait le bilan.

\section{TABLE 2 | BILAN DES GROUPES DE DISCUSSION}

\begin{tabular}{|c|c|c|c|c|c|}
\hline $\begin{array}{l}\text { District } \\
\text { Sanitaire }\end{array}$ & Localité & Groupe d'âge & Milieu & Nbre de GD & $\begin{array}{c}\text { Nbre de } \\
\text { participantes/ } \\
\text { GD }\end{array}$ \\
\hline \multirow{2}{*}{ Mbour } & \multirow{2}{*}{ Mbour } & $25-34$ ans & Urbain & 1 & 10 \\
\hline & & $35-44$ ans & Urbain & 1 & 10 \\
\hline \multirow{3}{*}{ Thiadiaye } & Thiadiaye & $25-34$ ans & Péri-urbain & 1 & 10 \\
\hline & Fissel & $35-44$ ans & Rural & 1 & 10 \\
\hline & Sandiara & $25-34$ ans & Rural & 1 & 10 \\
\hline \multirow{3}{*}{ Popenguine } & Popenguine & $35-44$ ans & Péri-urbain & 1 & 8 \\
\hline & Sindia & $35-44$ ans & Rural & 1 & 12 \\
\hline & Ndayane & $25-34$ ans & Rural & 1 & 10 \\
\hline Total & & & & 8 & 80 \\
\hline
\end{tabular}

\section{PERCEPTIONS SUR LA PF}

Les résultats des entretiens menés avec les acteurs PF, ainsi que les GD avec les femmes montrent que la PF est globalement perçue comme un domaine ayant connu beaucoup d'avancées ces cinq (5) dernières années.

\section{Perception des acteurs PF}

Selon les acteurs PF, le plus remarquable des changements positifs est l'élargissement du nombre et la diversité des acteurs intervenant dans le domaine. Pour plusieurs acteurs interrogés, c'est le fait que la pratique contraceptive soit désormais considérée non plus seulement comme un enjeu de santé mais comme un enjeu de développement qui a conduit à l'implication d'autres institutions étatiques en dehors du MSAS, comme les Ministères de la Jeunesse, de la Famille, et de l'Education Nationale. Comme l'indiquent ces propos d'un représentant d'ONG, "....les autorités administratives aujourd'bui répondent présent aux activités de PF. Il s'agit [aussi bien] des autorités au niveau central que décentralisées tels que les gouverneurs, les préfets qui président les réunions et mettent en place des comités de suivi comme l'exemple récent de la région de Saint-Louis où un comité régional de développement (CRD) a été présidé par le Gouverneur et un comité de suivi mis en place ».

Au-delà du leadership et des engagements de l'Etat, les acteurs ont également noté un meilleur engagement/implication des parlementaires, des OSC, des ONG et des PTF qui ont manifesté beaucoup d'intérêt à la PF à travers leurs appuis pour l'élaboration et la mise en œuvre du PANPF 2012-2015. 
"Aujourd'bui, il y a beaucoup plus de partenaires au développement qui s'intéressent un peu plus à la santé de la mère et de l'enfant [et] qui interviennent dans ce cadre-là. Et donc ça aussi c'est une bonne évolution, parce qu'avant cétait presque uniquement l'US AID qui intervenait et les gens se sont dit automatiquement " ab les américains, ils viennent pour limiter les naissances de nos enfants. » Et c'était la perception que les gens en avaient. Maintenant, bon comme ily a plusieurs partenaires qui interviennent. En principe, c'est mieux perçu, beaucoup mieux perçu» (Représentant ONG).

La PF apparait désormais comme un domaine mieux organisé, avec une vision, des stratégies et des plans de mise en œuvre. Elle fait l'objet d'une meilleure coordination grâce à la mise en place au niveau central d'un Comité Technique qui se réunit régulièrement et qui regroupe l'ensemble des acteurs, aussi bien Gouvernement que PTF, pour engager des discussions sur des questions techniques et financières. Ce cadre de concertation n'existait pas avant. Le Comité de Sécurisation des Produits SR (SPSR) assure lui aussi la coordination au niveau central. Il gère la quantification nationale, notamment avec les Tableaux d'Acquisition des Contraceptifs (TAC), et le suivi du tableau de bord qui permet de fournir les produits à tous les niveaux et de suivre l'approvisionnement par bailleur. «Avant on ne sentaitpas cette coordination, chaque partenaire essayait de travailler suivant un domaine et peut être qu'il n'y avait pas cette concertation entre les partenaires techniques et financiers... les différentes instances de coordination nous ont permis d'éviter les doublons dans les activités et de voir un peu les gaps " (Représentant du Gouvernement). De même au niveau régional, la mise en place des Comités Régionaux de Développement (CRD) et de Comités locaux de Développement (CLD), même s'ils ne sont pas réguliers, permet de réunir tous les acteurs, comme le fait remarquer cette autorité sanitaire locale: "Ici à Thiès, nous n'avons pas ce problème-là. Tous les acteurs sont impliqués dans le cadre des CRD et des CLD. Ce qu'il faut peut-être améliorer, c'est la régularité de ces moments de partage $»$.

Par ailleurs, certaines personnes, notamment au niveau régional, soulignent une meilleure adhésion, voire participation des communautés aux interventions de PF, plus spécifiquement les hommes et les leaders religieux. Cela est attribué essentiellement aux activités de communication avec le changement observé dans les discours et messages de sensibilisation consistant à mettre en avant l'importance de l'espacement des naissances à travers le slogan "Moytou nef». Le «Nef» [grossesse rapprochée] étant considéré sur le plan traditionnel et religieux comme un facteur d'épuisement de la femme.

"Maintenant avec le concept «Moytou nef 》 les gens ont mieux compris. La preuve en est qu'bier j'ai eu à capaciter des relais religieux car Popenguine a quand même cette spécificité. Dans le domaine communautaire nous sommes bien lotis. On a eu à capaciter dans la commune 23 relais religieux, des Imams et Oulémas sur l'argumentaire PF. Mais avec le concept «Moytou nef» ils ont émis des idées comme quoi si on avait dit ça avant il n'y aurait pas eu de problèmes. Eux ils disent que dans l'Islam, l'intervalle inter génésique dépasse au moins 2 ans » (Membre ECD).

Aujourd'hui, en plus de paraitre plus accessible aux populations aussi bien sur le plan financier que géographique, la PF est perçue comme un domaine qui dispose de ressources humaines de meilleure qualité grâce aux multiples formations dont elles bénéficient, et qui offre plus de choix aux femmes, même au niveau communautaire, du fait de l'élargissement de la gamme des méthodes.

Malgré toutes ces avancées, il reste des aspects à renforcer, de l'avis d'acteurs aussi bien au niveau national que régional. Ainsi, l'existence de détracteurs appartenant aux communautés religieuses constitue pour certains un obstacle à la hausse des indicateurs. Certains acteurs font aussi remarquer que le volet genre n'a pas suffisamment été pris en compte dans les efforts d'Information, Education et Communication (IEC) à l'endroit des populations, ce qui, selon eux expliquerait la réticence de certains hommes. D'autant plus que comme le souligne cet informateur : «En Afrique le powvoir économique est toujours détenu par l'homme, il est important qu'on puisse l'associer dans les prises de décisions. ... Tant que c'était les femmes qui s'adressaient aux hommes, les hommes ne les écoutaient pas. Moi je pense qu'il faut de plus en plus d'hommes qui s'intéressent à ce domaine. C'est extrêmement important que les hommes soient sensibilisés, qu'ils sachent qu'ils sont les premiers à tirer avantage de cette planification avant même la femme parce que si on 
a une femme qui est tout le temps malade, des enfants qui sont tout le temps malades, l'homme il est le premier à le sentir. Donc cette approche genre est extrêmement importante » (Représentant Gouvernement).

Par ailleurs, beaucoup pensent que malgré la baisse affichée des prix des contraceptifs, telle que décrite dans la revue documentaire, les services PF ne sont toujours pas accessibles à toutes les franges de la population, dans la mesure où en plus du prix du produit, tous les frais liés au service contribuent à les rendre hors de portée dans certaines zones. Un informateur témoigne : "Quand le produit est moins cher et que l'acte globalement du prestataire est cher, l'acte va noyer la faisabilité. Prenons juste la pose d'un implant qui va nécessiter de la xylocaïne, des compresses, tous ces intrants qui concourent à cet acte vont être chers alors que le seul produit à insérer coûte dans l'ordre de 500 francs» (Représentant ONG).

Les difficultés à intégrer les données du secteur privé (cliniques, cabinets médicaux privés, pharmacies, services médicaux d'entreprises), qui constituent de l'avis de beaucoup d'acteurs une réelle potentialité pour booster le TPC, participeraient à renforcer la perception négative de la PF. Beaucoup pensent d'ailleurs que les données d'utilisation de la PF sont sous-estimées, ce qui pose la question de leur fiabilité. Les propos suivants illustrent ce constat : "Je viens d'un audit de la qualité des données. Par exemple rien que pour le cas de Dakar, où il y a beaucoup plus de structures privées que de structures publiques, pratiquement les données du secteur privé ne sont pas dans nos données. Donc pour dire que les données que nous avons sont sous-estimées, heureusement qu'il y a l'EDS Continue qui nous permet un peu d'avoir une estimation du taux de TPC. Mais nous devons nous battre pour avoir un système de collecte, de remontée de données fiables, ça je pense que c'est une faiblesse du programme » (Représentant du Gouvernement).

En outre, pour beaucoup d'acteurs aussi bien au niveau central que régional, le maintien des femmes dans le programme mérite une attention particulière au regard des nombreux cas d’abandons relevés.

Un autre aspect qui a été souligné et qui est également perçu comme une faiblesse du programme concerne l'insuffisance du personnel qualifié, situation qui a engendré le recours au personnel communautaire dont la motivation constitue jusqu'à présent une préoccupation. Les propos de ce représentant d'ONG le confirment : "Ils sont là mais il y a beaucoup de bénévolat dans la santé, beaucoup de bénévolat. Et on ne peut pas demander des résultats à des gens qui en retour posent le problème de motivation. Il y a ce problème».

Par rapport à la sécurisation des produits, certes l'Etat fournit des efforts considérables pour améliorer leur disponibilité, cependant, le budget affecté par le Gouvernement à la PF est jugé insuffisant par beaucoup d'acteurs.

Par ailleurs, et bien que la DSRSE essaie de fédérer les actions, la synergie demande à être renforcée car certains acteurs constatent toujours un manque de coordination des interventions, occasionnant parfois des doublons et des télescopages dans les activités.

\section{Perception des femmes bénéficiaires}

Du côté des femmes bénéficiaires, la PF est également perçue comme une pratique ayant subi des changements essentiellement positifs. En effet, elles soulignent un réel engouement des femmes et une adhésion massive à son utilisation, ce qu'elles attribuent à plusieurs facteurs, notamment :

- L'augmentation des connaissances sur la PF : la plupart des femmes pensent que les populations ont une meilleure connaissance sur les avantages et inconvénients grâce à l'intensification de la sensibilisation et des actions de communication. De ce fait, elles comprennent mieux les enjeux de la PF. 
"Les femmes comme moi avaient peur de faire la PF. Mais on les a tellement sensibilisées que maintenant, la plupart d'entre elles n'bésitent plus à aller à l'hôpital pour faire la PF, parce qu'on avait dit "si vous faites la PF, avant d'avoir un enfant à nouveau, vous serez confrontées à beaucoup de difficultés". Mais on nous a sensibilisées par rapport à tout cela. On dormait avant, mais maintenant, nous sommes éveillées par rapport à ça. » (GD Fissel).

Ou selon cette autre participante : "Des femmes comme moi avaient des grossesses très rapprochées mais depuis ce n'est plus le cas. Les grossesses sont espacées et c'est une bonne chose. Les femmes sont maintenant éveillées. Auparavant on ne savait rien. Les grossesses rapprochées nous fatiguaient, mais maintenant Dieu merci « Alhamdoulilah » (GD Mbour).

- L'accessibilité financière : la réduction du coût des méthodes contraceptives a, selon certaines femmes, beaucoup contribué à l'accroissement de leur utilisation.

"Auparavant, tu voyais quelqu'une qui disait "je ne peux pas y aller car je ne dispose pas d'argent". Mais actuellement on a réduit tous les coûts, on peut dire que c'est presque gratuit. Les produits PF qui coûtaient $500 \mathrm{~F}$ auparavant, coûtent $200 \mathrm{~F}$ actuellement, on le fait à vil prix maintenant » (GD Fissel).

- L'augmentation des types de méthodes disponibles au sein de la gamme: la diversité des méthodes contraceptives incluses dans la gamme a augmenté le choix des femmes et permet à chacune d'opter pour la méthode qui lui convient.

Par ailleurs, l'usage de la PF est également perçu comme un facteur contribuant à la réduction des grossesses rapprochées, au bien-être et à l'épanouissement des femmes. Mieux, les participants constatent qu'en espaçant les naissances, la PF constitue la raison principale de la baisse des décès maternels.

"Quand il n'y avait pas la planification familiale, les grossesses étaient rapprochées. Avant que ton enfant atteigne un an, tu tombes enceinte. Elle sert à espacer les naissances. Tu peux rester 2 ans avant de retomber enceinte. Si tu le désires aussi, tu peux rester 3 ans avant de prendre une autre grossesse. C'est vraiment bien 》(GD Ndayane).

"Nous saluons le planning car il nous a beaucoup arrangé nous les femmes. Les décès maternels et néonataux ont diminué et la PF y est pour quelque chose. Auparavant la femme mourrait en accouchant mais avec le planning cela n'existe plus. Les naissances sont espacées et tu as le temps de t'occuper de tes enfants. V raiment le planning nous arrange beaucoup» (GD Popenguine).

«Maintenant, c'est rare de voir une femme qui meurt en accouchant 》(GD Popenguine).

\section{CONNAISSANCE DE L'APPROCHE 3D}

Les informations recueillies dans le cadre des entretiens approfondis montrent que l'approche 3D n'est connue que d'environ 1/5ème des acteurs intervenant dans le domaine de la PF. Cette méconnaissance est encore plus notoire dans la région de Thiès où la quasi-totalité des acteurs interrogés reconnait en avoir entendu parler pour la première fois à l'occasion de la présente étude. Cette ignorance serait liée, selon un des répondants, au manque de communication autour de l'approche.

Parmi les rares acteurs PF qui déclarent en avoir entendu parler, à l'occasion du lancement du PANPF pour certains ou lors d'une réunion du Comité Technique PF pour d'autres, très peu ont pu en donner une définition exacte, ou même des informations relatives à sa date d'apparition et à ses potentielles applications dans des domaines autres que la PF. Toutefois, une source indique que cette approche avait été énoncée pour la première fois en 2010 à Kigali, lors d'une réunion sur la PF, donc avant la Conférence de Ouagadougou de 2011 à laquelle les acteurs et les documents retrouvés pour la revue documentaire font référence. De plus, quelques-uns ont souligné qu'en dehors de la PF, elle est appliquée dans d'autres domaines de la santé tels que la santé maternelle, 
néonatale et infantile (dans le cadre notamment du Programme Elargi de Vaccination (PEV), de la nutrition, de l'utilisation de la ventouse obstétricale dans les postes de santé) et le paludisme.

Le constat reste le même du côté des femmes bénéficiaires en ce qui concerne le manque de connaissance de l'approche 3D. En effet, toutes les participantes ont déclaré n'avoir jamais entendu parler de cette approche.

\section{CONNAISSANCE DES ACTIONS MENEES DANS LE CADRE DE CHAQUE " D "}

\section{DEMOCRATISATION}

Bien que peu de personnes interrogées connaissent l'approche 3D, beaucoup parmi elles ont cité des actions qui ont été menées dans le cadre de la "Démocratisation » à une fois que la définition du concept leur a été donnée. La recherche qualitative confirme ce constat. Les actions reconnues sont décrites dans la revue de la littérature.

\section{Actions citées par les acteurs PF}

\section{Actions menées sur le plan politique, légal et règlementaire}

Beaucoup d'acteurs ont attiré l'attention sur l'engagement des autorités à faire de la PF une question prioritaire et sur la réelle volonté politique de rendre les services accessibles. C'est ainsi qu'un membre d'ECD de la région de Thiès affirme : "Ca on l'a senti parce que pendant des années c'était uniquement les agents de la santé qui parlaient de la PF, mais si maintenant le Président, le Ministre et tout le monde en parlent, c'est qu'il y a la volonté politique ». Et cette parlementaire de confirmer «Ma première action a été d'organiser un séminaire de sensibilisation sur la planification familiale ».

Cette volonté politique s'est manifestée à travers plusieurs actes, tels que la création de la DSRSE avec en son sein la DPF (Cf. Revue documentaire pour plus de détails). De plus, d'autres secteurs/acteurs ont été impliqués dans les questions de PF et des outils de plaidoyer ont été élaborés, comme l'outil ENGAGE, pour les sensibiliser à l'apport de la PF aussi bien dans l'amélioration de la santé des mères, des nouveau-nés et des enfants mais aussi dans la lutte contre la pauvreté.

L'Etat s'est également engagé sur le plan budgétaire, notamment par l'annonce du Ministère d'augmenter de 200 pour cent la ligne budgétaire dédiée aux contraceptifs. Et depuis lors, beaucoup d'acteurs déclarent avoir constaté que des efforts ont été faits dans ce sens par le MSAS, mais aussi par le Ministère de l'Economie et des Finances, comme l'indique un représentant du Gouvernement : "On était parti de 50 millions, après c'est passé à 100 millions puis à 150 millions, maintenant on est à 200 millions et on risque de le porter à 300 millions. Et donc même le plaidoyer va continuer ». Ces propos sont corroborés par ceux d'un autre représentant du Gouvernement: "Je crois que la ligne était avant à 65 millions, ensuite on est allé à 100, ensuite à 200 millions, en 2016 la ligne sera à 300 millions. Aujourd'bui quand je suis sorti de la réunion tenue au Ministère de l'Economie et des Finances, je sais que par exemple on va augmenter pour la DSRSE encore une ligne comme contrepartie avec le système phare et le financement direct avec l'US AID. Donc, vraiment ily a des efforts que le gouvernement est en train de faire autour de ça. Je pense que c'est une bonne chose ».

Par ailleurs, les Politiques, Normes et Protocoles (PNP) sont révisées de manière régulière, pour permettre de réactualiser les décisions. Aussi, le Sénégal s'est doté d'un plan de sécurisation des produits d'importance vitale pour la santé de la mère et de l'enfant, dont les produits contraceptifs font partie en tant que médicaments essentiels. Sa mise en œuvre implique de nombreuses initiatives des partenaires. Un représentant d'ONG affirme : "Au début les produits contraceptifs étaient gérés directement par la DSRSE. Maintenant, il y a eu un changement majeur, c'est l'intégration des produits contraceptifs dans les médicaments essentiels... Maintenant, avec l'intégration des contraceptifs 
dans les médicaments essentiels, ça a aussi démocratisé quand même. Le fait de considérer les produits contraceptifs comme des médicaments essentiels ça participe à la démocratisation ».

Egalement, l'élaboration du plan stratégique de santé communautaire, qui date de 2014, a été mentionnée par certains acteurs comme la politique qui a commencé à définir cette démocratisation au niveau communautaire et a permis d'établir un cadre législatif et réglementaire pour les interventions. Cela apparait à travers les propos de ce représentant d'ONG : "J'ai vu que la politique de santé communautaire a beaucoup amené à l'accès des services dans le faire faire. Et le terrain nous l'a prouvé. Aujourd'bui aux alentours de tous les centres de santé, nous sentons une mobilisation communautaire qui permet d'accéder aux services $P F »$.

Les acteurs du niveau régional ont beaucoup insisté sur l'extension des infrastructures sanitaires, notamment la répartition des postes de santé tous les cinq (5) km, d'autant que l'option stratégique dans la région de Thiès a été de faire en sorte que tous les postes de santé soient doublés en sages-femmes, en renfort aux Infirmiers Chefs de Poste (ICP).

Par rapport à l'offre de services, de nombreux acteurs affirment que démocratiser, c'est aussi garantir des ressources humaines de qualité. De ce fait, les nombreuses formations initiées à l'endroit du personnel de santé, avec un accent particulier sur le counseling pour faciliter le choix éclairé des femmes, auraient beaucoup contribué à la démocratisation. Un acteur PF au niveau régional affirme : "Actuellement, le personnel également est mieux formé sur la PF grâce aux nombreuses formations offertes sur les différentes méthodes, de sorte que les clientes peuvent aujourd'hui faire un choix éclairé ». Et cet autre concourt : "Très souvent des formations sont organisées pour le personnel de santé car la médecine évolue et nous avons de plus en plus de nouvelles méthodes qui intègrent la gamme comme récemment avec le Sayana ${ }^{\circledR}$ Press, et on parle même d'une nouvelle méthode, l'anneau vaginal, qui va bientôt rejoindre la gamme».

\section{Actions de communication}

Selon les acteurs interrogés, parmi les activités qui ont le plus démocratisé les services de PF figure la campagne nationale de communication « Moytou nef » qui a été initiée dans le cadre de la mise en œuvre du PANPF (Cf. Revue documentaire). De leur point de vue, elle demeure l'activité de communication qui a le mieux touché les différentes cibles, notamment les hommes et les leaders religieux. Les campagnes de sensibilisation menées à travers les médias (télés, radios locales) et les affiches ont permis aux populations d'avoir un regard nouveau sur la PF et de mieux y adhérer. Ainsi, des associations et réseaux de religieux constitués d'Imams et d'Oulémas ont été chargés, après avoir été formés, de sensibiliser à leur tour à travers des prêches au niveau des mosquées et des quartiers, et auprès des hommes. Un membre de l'ECD de Mbour témoigne: "Les religieux sont entrain de bien nous aider par rapport à la sensibilisation sur la planification familiale surtout au niveau des hommes ». Et un autre à Popenguine confirme : "Avec le réseau des "relais religieux", comme on les appelle dans notre district, nous avons tenu même une formation avant-bier dans le cadre de l'argumentaire religieux sur la planification familiale. Donc eux aussi, ils jouent un rôle qui est assez. important parce que là où je ne peux pas connaître les « sourates » ou un "ayâ » pour pouvoir convaincre quelqu'un, eux, il y a certains gens à qui ils peuvent tenir ce langage et qui comprennent....avec eux, il y a quand même de plus en plus une prise de conscience des gens qui étaient réticents au début et qui commencent quand même à accepter».

\section{Actions ayant des effets sur le coût}

Pour la plupart des acteurs, la circulaire sur les prix émise par le Ministère de la Santé en 2010, et en principe affichée au niveau des structures sanitaires, constitue un bon exemple de démocratisation, dans la mesure où elle a permis d'harmoniser et de réduire les prix des contraceptifs (Cf. Revue documentaire). Suite à cette disposition, la participation des clientes est devenue symbolique, et elle est théoriquement la même à tous les niveaux de la pyramide sanitaire. Les propos de ce membre de l'ECD de Thiadiaye sont assez illustratifs : «Ily a une harmonisation du prix des produits. Ce qu'on constatait par exemple pour certains produits, c'est qu'un district pouvait vendre à $1000 \mathrm{FCFA}$, un autre à $1500 \mathrm{FCF}$, et un autre à $500 \mathrm{FCFA}$. Maintenant tous les districts, tous les points de prestation sont tenus de respecter le prix ; le plus cher produit est à $500 \mathrm{FCF} A$ ». 
Certains intervenants tels que Marie Stopes International (MSI) seraient même allés plus loin en rendant le service gratuit à travers certains canaux d'offre de services telles que les équipes mobiles, ce qui veut dire que la baisse du prix des contraceptifs, ou gratuité dans certains cas, combinée au rapprochement des services, rend quasiment nul le coût des services dans certaines zones.

"Sur le plan financier également, il y avait les déplacements parce que pour obtenir certaines méthodes de planification familiale, les populations étaient obligées de venir jusqu'au niveau du centre de santé, maintenant au niveau du poste de santé le plus proche, elles peuvent accéder à cette planification familiale, donc moi je pense que sur le plan financier, le coût a vraiment diminué pour la population pour ne pas dire que le coût est inexistant, par rapport à certaines activités telles que les cliniques mobiles et autres qui offrent gratuitement les méthodes et qui vont vers les populations et qui réduisent le coût du transport et autres 》 (Autorité sanitaire Mbour).

\section{Leadership et Autonomisation des femmes}

En championne de la PF, Madame la Ministre de la santé est, de l'avis de certains acteurs, un bon exemple de leadership des femmes. En outre, le fait que les femmes parlementaires soient de plus en plus engagées sur les questions de PF, contribue à rendre le leadership plus fort. Ce partenaire technique informe d'ailleurs que "Certaines parmi elles ont d'ailleurs participé à la dernière réunion du Partenariat de Onagadougou à Paris ». Par ailleurs, pour magnifier le leadership féminin, en dehors du constat selon lequel «les réseaux d'acteurs communautaires sont à 90 pour cent constitués de femmes ", d'autres informateurs mettent en exergue l'existence de réseaux de femmes qui soutiennent/accompagnent le MSAS dans le cadre du plaidoyer. Ce représentant du Gouvernement en témoigne : "Dans le domaine de la PF également ily a des réseaux de femmes, qui sont très actifs et qui nous aident beaucoup. Je veux parler des femmes médecins, l'Association des Femmes Médecins (AFEMS), le Réseau Siggil Jigeen (RSJ) constitué de femmes et qui appuie beaucoup le Ministère de la Santé, l'Association des femmes juristes (AJS) qui aide beaucoup le Ministère de la Santé dans le domaine du plaidoyer. Je pense qu'en termes de leadership féminin, les femmes sont beaucoup impliquées dans la $P F \gg$.

Mais pour beaucoup d'acteurs, c'est avec le Programme «Bajenu gox » (Cf. Revue documentaire), qui a été redynamisé ces dernières années, que le leadership s'est vraiment affirmé à travers la sensibilisation auprès des hommes (et des belles-mères), détenteurs du pouvoir de décision au sein des ménages. Une source affirme : «Je pense que les bajenu gox ont été aussi utiles par rapport à la promotion de la planification familiale. Et ce qui est intéressant à noter, c'est que ce leadership féminin là, ça a concouru à renforcer l'implication des hommes. Parce que le problème ce n'étaient pas les femmes en réalité ».

Concernant l'autonomisation des femmes, même si certains informateurs pensent que ce n'est pas encore le cas, d'autres font remarquer que les nombreuses sensibilisations menées au sein des organisations féminines contribuent à rendre les femmes moins dépendantes vis-à-vis de leur mari, notamment en ce qui concerne la prise en charge de leurs besoins en santé. La promotion des activités économiques au sein de ces instances, ainsi que l'avènement des mutuelles de santé, facilitent de plus en plus leur autonomisation. Les propos de ce partenaire technique en sont une bonne illustration: "Avec les nombreuses sensibilisations qui se font au niveau des associations et groupements féminins, les femmes ont désormais compris qu'elles sont en mesure de prendre elles-mêmes en charge leur propre santé, et notamment en matière de PF. Traditionnellement, la femme dépendait de son mari pour garantir sa santé. Or, aujourd'bui avec les activités génératrices de revenus dans lesquelles beaucoup de femmes s'activent, elles peuvent se prendre en charge elles-mêmes sans avoir besoin de passerpar le mari. Donc elles sont de plus en plus autonomes ». Ces propos sont corroborés par ceux d'un membre de l'ECD de la région de Thiès : "Même dans leurs activités les gens se sont organisés. C'est avec les activités génératrices de revenus. Je sais que je peux me prendre en charge, je peux ne pas dépendre de mon mari, je peux en même temps prendre en charge ma santé et la santé de mes enfants. Ça en tous cas la sensibilisation y est. Nous y sommes. Le changement est toujours de longue durée, on ne peut pas le mesurer aussitôt, mais quand même, on sent que ça bouge». 


\section{Actions en faveur des jeunes}

Même si le PANPF cible spécifiquement les jeunes en union, les acteurs ont pu relever certaines actions dans le cadre de la démocratisation des services de PF à l'endroit de cette cible de façon générale, parmi lesquelles :

- La création au sein de la DSRSE d'une Division pour la SR des adolescents/jeunes (SRAJ).

- L'élaboration d'un Plan Stratégique de la Santé des Adolescents/Jeunes.

- La mise en place au niveau de certaines structures de santé, notamment au niveau régional, d'un Espace jeune/Coins Ados où ils peuvent avoir accès à l'information liée à la SR ou être orientés. Cela, non seulement pour être conforme aux "Standards des Services de Santé Adaptés aux Adolescent(e)s/Jeunes », notamment le standard 2 (Cf. Revue documentaire), mais surtout pour la prise en charge spécifique de cette catégorie de population. Un représentant du Gouvernement fait remarquer que : "Dans chaque région, il y avait un Centre Conseil Ados. Mais nous, au niveau du département ministériel nous avons pensé que ce n'était pas démocratique. On n'a pas le droit de ne donner cette chance qu'à des résidents des chefs-lieux des régions. Et qu'il fallait peut être, la meilleure façon c'est de s'ouvrir aux postes de santé, d'avoir un petit coin là-bas, un espace où on met un petit équipement pour poser des discussions entre pairs, leur permettre en tout cas de poser toutes les questions et d'avoir l'offre de service qui est disponible ».

- La mise en place, avec l'appui de partenaires, de réseaux et associations de jeunes qui sont très impliqués dans la PF (Ex : réseau des jeunes ambassadeurs de la PF, RESOPODEV, etc.).

- La formation de pairs éducateurs pour la sensibilisation des jeunes sur les questions de SR parmi lesquelles la PF : "On les a choisis dans chaque village. Dans chaque village il y a un représentant qui parle aux jeunes. S'ily a des activités ils se rassemblent pour avoir plus de force et d'écoute » (Membre ECD Popenguine).

Certains informateurs insistent sur les stratégies avancées de partenaires à l'endroit des étudiants, comme par exemple la mise en place de centres/espaces jeunes dans certaines universités, qui sont parfois perçus par quelques acteurs comme des cliniques mobiles comme en témoigne ces propos : "Il y a MSI également qui nous aide beancoup avec les cliniques mobiles au niveau de l'UCAD [Université Cheikh Anta Diop] et an niveau de l'Université Gaston Berger. Ils font un excellent travail dans ce domaine » (Représentant du Gouvernement).

\section{Partenariat Public-Privé}

L'étude montre que dans le cadre du Partenariat Public-Privé (PPP) à but commercial, de nombreuses stratégies ont été mises en place pour démocratiser la PF à travers l'implication du secteur privé dans l'offre des services. La plupart des acteurs qui se sont prononcés sur cette question ont fait part de l'existence, depuis fin 2012, d'un réseau de franchises sociales appelé "Blue Star" créé dans le cadre du projet ISSU avec l'intervention des membres du consortium, notamment MSI, et qui a pour but d'impliquer les structures de santé privées dans l'offre de services PF (Cf. Revue documentaire). Ces structures bénéficient d'appui sur le plan technique (formations), matériel et logistique. Au niveau régional, des interventions sont également menées dans les districts sanitaires en partenariat avec des structures de santé privées. Un membre de l'ECD de Thiès affirme : "Dans la région de Thiès nous avons établi un partenariat avec le secteur privé afin qu'il soit mieux impliqué dans l'offre des services PF. Dans ce cadre, ils bénéficient des mêmes formations que nous offrons au personnel du public, ils sont invités dans les réunions et ils reçoivent les produits dans les mêmes conditions que le secteur public à travers le Push Model».

Il est également noté le travail mené par MSI avec un réseau de 82 pharmacies pilotes, toujours dans le cadre du projet ISSU2. Cela a permis d'intégrer la PF à leurs rayons, de promouvoir la demande et de suivre la distribution grâce à la collecte de données.

2 Ce réseau est constitué à Guédiawaye, Pikine, Keur Massar et Mbao pour faire le counseling, le marketing et le référencement des clientes auprès des structures habilitées. 
En plus des cliniques privées, l'implication des services médicaux de grandes entreprises (SENELEC, SONATEL, SONES) dans le cadre de l'initiative Informed Push Model (IPM), à travers la contractualisation, a également été mentionnée par certains acteurs. En outre, beaucoup de structures privées du district sont enrôlées dans le cadre de l'initiative du Tutorat Plus (Cf. Revue documentaire).

Tous convergent vers l'idée que l'objectif de toutes ces interventions, au-delà de faciliter l'accès aux services de $\mathrm{PF}$, est de collecter les données du secteur privé en vue de les intégrer à celles des districts sanitaires.

Toujours dans le domaine du PPP, hormis l'offre de services, certaines sources gouvernementales informent de la collaboration avec des fondations (Ex : Fondation SONATEL) pour la mobilisation de ressources pour la PF. Des démarches seraient aussi prévues pour un partenariat avec le secteur minier, en vue de les impliquer dans la PF.

En ce qui concerne le PPP avec le secteur privé à but non lucratif, certaines sources indiquent que beaucoup de choses ont été faites, telle que la mise en place de la Stratégie Nationale de Contractualisation des Services de Santé et de son plan d'opérationnalisation. De même, avec la mise en place de la Stratégie Nationale de Santé Communautaire, le Gouvernement a su impliquer ce secteur. Ce représentant d'ONG souligne que : «Récemment le Ministère de la Santé a mis en place une stratégie nationale de santé communautaire. Et tout ceci c'est fait avec les partenariats, avec les organisations non-gouvernementales, avec les autres secteurs qui ne sont pas lucratifs mais qui sont privés. Une ONG est une structure privée, mais à but non lucratif. De ce point de vue, je vois qu'il y a beaucoup d'ouvertures qui existent. Il y a un grand partenariat, parce qu'il y a de grands programmes qui sont financés par l'US AID qui viennent apporter un soutien au programme. Et ça je crois qu'ily a des ONG sénégalaises qui sont là ».

\section{Actions citées par les femmes bénéficiaires}

Les femmes ont, pour la plupart, essentiellement retenu deux (2) actions de ce qui a été fait durant ces cinq (5) dernières années pour démocratiser les services de PF.

Il s'agit d'abord des activités de sensibilisation menées à travers les télés ou radios (y compris les radios communautaires) dans le cadre de la campagne «Moytou nef» ou par les relais et bajenu gox dans le cadre des visites à domicile. Ces activités ont pour but, selon elles, d'améliorer les connaissances des populations sur la PF, notamment des hommes, avec l'appui des leaders religieux.

"Les télés montrent beaucoup de choses à ces sujets, elles parlent même sur la planification familiale. Elles collaborent maintenant avec les Imams pour améliorer l'accès à la planification familiale. Je l'ai vu personnellement à la télé. C'est l'Imam qui est à Sud FM. Comment il s'appelle encore....il parle beaucoup sur la planification familiale. Je l'ai vu personnellement. Il disait qu'au début il était détracteur de la planification familiale, mais après avoir bien réfléchi, il s'est dit que la planification familiale n'est pas manvaise » (GD Ndayane).

"A propos du planning on parle tout le temps de "Moytou nef». Les radios, les télés en parlent. Même celles qui ne sont pas instruites savent ce que c'est qu'espacer les naissances » (GD Mbour).

"Avec la démocratisation, même les hommes sont réveillés parce que ce sont eux-mêmes qui rappellent à leurs épouses leurs rendezvous. Ce sont eux qui disent "c'est quand ton rendez-vous?" Ou "as-tu pris tes pilules?" Ils sont éveillés maintenant. ... ils font plus d'économies car si tu as un bébé, 3 mois après tu tombes encore enceinte tu n'es pas en bonne santé, ton enfant est malade, ton mari n'aura pas de moyens » (GD Mbour).

L'autre action qui a été menée et qui a, selon la plupart des femmes, contribué à la démocratisation, est la baisse des prix, voire la gratuité dans certains cas, des services PF. 
"La démocratisation signifie qu'auparavant tu payais $1000 \mathrm{FCF}$ A pour faire la planification, maintenant c'est gratuit, tu vois que c'est démocratisé» (GD Mbour).

"J'ai vu que la planification est utile, on l'a démocratisée c'est-à-dire c'est gratuit pour que tout le monde puisse y accéder, aussi bien celles qui ont les moyens que celles qui n'en disposent pas. Bref on l'a démocratisé » (GD Mbour).

De plus, une source fait remarquer que le fait pour les autorités de considérer la pratique contraceptive comme un droit humain représente une action démocratique en soi.

"C'est le fait que les femmes mariées, comme les femmes non mariées, toutes les femmes qui sont en âges de procréer et qui soubaitent faire la planification y ont droit sans qu'on leur pose de questions, ce sont les mesures prises par les autorités 》(GD Mbour).

\section{Succès et réalisations de la démocratisation}

De nombreux succès et réalisations ont été associés à la démocratisation.

Pour les acteurs $P F$, il s'agit notamment de :

- Meilleure perception de la PF par les populations.

- Meilleure connaissance des populations de la PF (parce que mieux informées)/Meilleur accès à l'information.

- Meilleure implication des autres secteurs (privé commercial et privé à but non lucratif) dans les interventions en matière de PF.

- Accès plus facile et équitable (sur le plan financier) de la population à la PF.

- Meilleure implication du niveau communautaire. Un représentant du Gouvernement indique: «Ils se sont rendus compte que ce n'est plus l'affaire du secteur de la santé mais c'est plus l'affaire de la communauté. Parce que la plupart des agents ne sont pas rémunérés donc ils le font à titre bénévole, et ça, ça demande un engagement. Ça veut dire qu'au moins ils ont adhéré à la cause, donc la sensibilisation a été bien faite. Ils sont vraiment sensibilisés et ils se sentent concernés. Donc ça également c'est des facteurs de succès ».

- Elargissement de l'offre.

- Début d'intégration/prise en compte des données des autres secteurs.

- Mise à jour des programmes pour le privé à travers les formations reçues.

- Amélioration de l'autonomisation des femmes.

Pour les femmes bénéficiaires, les succès de la démocratisation sont:

- Amélioration des connaissances des populations.

- Utilisation accrue de la PF par les femmes.

- Accessibilité financière aux services de PF.

- Espacement des naissances.

- Meilleure implication et adhésion des hommes.

"Actuellement ce sont les hommes qui imposent à leurs femmes d'aller faire la PF. Parce que j'ai une voisine que je taquinais souvent en lui disant "B.B.G : Boote, Biir, Gaddou" [porter sur le dos, porter dans le ventre, porter sur le bras]. Mais lorsqu'elle a accouché, son mari s'est déplacé jusque chez. moi Lorsqu'il est arrivé, il m'a demandée "qu'est-ce que je peux faire pour que ma femme fasse la PF ?" Je lui ai dit "vas à l'hôpital". Il me dit "accompagnes-la s'il te plait"... Actuellement, beaucoup d'hommes ont imposé à leurs femmes de faire la PF parce que la façon dont elles s'occupent d'eux leur fait plaisir »(GD Fissel). 
"Le fait que nos maris l'acceptent aussi fait partie des choses qui l'ont démocratisée. Si les chefs de famille sont d'accord, elle devient plus répandue. C'est mieux de te demander de partir que d'essayer de te retenir. Ils font partie des personnes qui ont démocratisé les services $P F \gg(G D N d a y a n e)$.

\section{Limites de la démocratisation}

Malgré les efforts fournis pour démocratiser les services PF, il reste, selon les personnes interrogées, certains aspects à améliorer.

\section{Pour les acteurs PF:}

- L'implication insuffisante du secteur privé, notamment les pharmacies, dans l'offre de service PF : actuellement, la pleine participation/implication du secteur privé, qui présente de réelles opportunités en termes de couverture, est entravée des textes juridiques restrictifs.

"Pour une réelle implication du secteur privé, il faut réviser ces textes dont certains constituent des barrières pour l'offre. Si on prend le cas de l'offre de services, nous avons eu à accompagner le Ministère sur certaines initiatives telles que l'offre initiale de pilules au niveau communautaire mais an niveau des pharmacies ily a toujours des blocages malgré le fait que la pharmacie soit une entité présente au niveau des communautés.... Si cela se fait au niveau des cases avec une bonne supervision, pourquoi pas au niveau de la pharmacie? Je pense qu'on peuty aménager un espace pour le counseling. Les agents des pharmacies demandent qu'on les forme pour offrir la PF. Au niveau des cases, ce ne sont pas toutes les méthodes qui sont offertes, donc pourquoi ne pas envisager l'extension de cette expérience au niveau des pharmacies?... Je pense que si on les met autour d'une table (DSRSE, secteurprivé etpartenaires clés), on pourra discuter de la faisabilité de certaines interventions au niveau du secteur et des contraintes de ce secteur, de leur implication par rapport aux nouvelles politiques du Ministère en matière de SR pour améliorer leurparticipation.» (Représentant ONG).

"Il reste beaucoup de choses à faire car le privé ne se sent pas encore comme faisant partie du système de santé. Or les privés doivent se sentir comme des parties prenantes, et seule une volonté politique peut le faire» (Partenaire technique).

- Le manque d'application de la circulaire sur les prix : pour de nombreux interviewés, malgré la baisse du prix des produits contraceptifs, en réalité sur le terrain, le coût du service reste encore élevé pour certaines clientes à cause des frais additionnels liés à l'offre de service par les prestataires.

"Même si le produit en lui-même il est accessible, s'il faut du coton ou une seringue ou bien autre chose, c'est la cliente qui paie et en fin de compte cela lui revient très cher » (Membre ECD Thiès).

"Ily a quand même quelque part la résistance des prestataires. Par exemple, les femmes nous disent souvent "oui, vous dites que ça coûte 500 francs. Mais pour mettre l'implant il faut du coton, compresse, etc. Mais, la sage-femme nous dit qu'il nous faut payer 1000 francs"... J'avais demandé aux partenaires de me donner des kits d'insertion et de retrait. C'est-à-dire un package où ily a tout là-dedans. Donc, il n'y aura plus d'bistoire... Je continue en tout cas à faire le plaidoyer. Ça je pense que c'est quand même une limite à ce qu'on est en train de faire » (Représentant du Gouvernement).

"Ily a le prix du produit, mais on rajoute aussi pas mal d'autres frais liés au service et ça donne des montants qui quelquefois ne sont pas accessibles pour tout le monde...Mais le contrôle jusqu'au niveau de la prestation, je pense que ça c'est un gros challenge. C'est quelque part le nerf de la guerre. Si on powvait agir là-bas ce serait bien, ce serait vraiment démocratique » (Représentant ONG).

- La réticence de certains hommes et leaders religieux : bien que ces cibles adhèrent de plus en plus à la PF grâce à la communication accrue à leur endroit, certains acteurs soulignent que quelques-uns restent réticents. 
"Il y a encore la réticence des religieux. A ce niveau ily a encore des choses à faire. Il faut les sensibiliser davantage. De même que certains hommes puisque nous avons nos réalités. Ici, c'est l'homme qui a le pouvoir de décision, et généralement ce sont eux qui prennent en charge les dépenses de santé. Mais aussi les belles-mères car quand les hommes ne sont pas là, ce sont elles qui décident » (Membre ECD Thiès).

- L'offre limitée de services à l'égard des jeunes : malgré ce qui a été fait à l'endroit de cette frange de la population, de l'avis de beaucoup d'acteurs, l'offre de services PF aux jeunes reste encore limitée par certains facteurs, notamment d'ordre socio-culturels, et législatifs, tel que le manque de décret d'application de la loi SR.

"Ce qui n'a pas bien marché c'est par rapport à la contraception des jeunes compte tenu des barrières culturelles comme je vous l'ai dit. Compte tenu de beaucoup de pesanteurs. Aussi l'Etat ne veut pas trop s'avancer sur ça. Donc c'est des points qui sont des défis pour la démocratisation. Parce qu'aussi chaque societé a sa culture, a ses normes sociales »(Représentant ONG).

- L'insuffisance de la participation financière de l'Etat : même si des efforts ont été constatés, la participation de l'Etat dans le financement de la PF est jugée minime par certains acteurs. Ces derniers pensent aussi que l'implication effective des autres secteurs serait une bonne chose (ex : Ministères de l’Economie, de la Jeunesse, de la Famille...).

"Le fait de mettre les produits contraceptifs dans le circuit des médicaments essentiels au même titre que les autres médicaments, c'est une excellente chose. Maintenant, ce qui reste c'est juste le financement par l'Etat. C'est peut-être la participation financière de l'Etat par rapport à la sécurisation des médicaments qu'il faut améliorer » (Représentant ONG).

\section{Pour les femmes bénéficiaires:}

Le principal aspect qui pourrait être considéré comme une limite de la démocratisation concerne l'offre de services aux jeunes. Alors que pour les acteurs PF cette offre doit être renforcée, pour la majorité des femmes, elle est considérée comme un excès de démocratisation, dans la mesure où le fait de trop vouloir démocratiser pourrait inciter les jeunes à la débauche.

"Ce qui n'a pas marché, ce n'est qu'une chose, j'ai remarqué qu'on offre des méthodes de PF aux jeunes filles, ça ce n'est pas normal. Une fille qui n'est pas mariée, on ne doit pas pouvoir lui offrir une méthode. La démocratisation doit se limiter aux femmes qui sont mariées, qui gèrent leur ménage, qui gèrent leur époux, qui gèrent leurs enfants. Mais une jeune fille qui va demander une méthode de PF, je ne suis pas d'accord sur cela 》 (GD Fissel).

"La démocratisation est importante mais ma préoccupation ce sont les jeunes qu'on y a intégrés, je croyais que la planification familiale était réservée aux femmes mariées, celles qui sont fatiguées d'enfanter et veulent arrêter et non aux jeunes filles non mariées ou bien ?...ça me dérange quand même, si ça ne tenait qu'à moi la planification familiale serait réservée aux adultes. Si les jeunes soubaitent en pratiquer bon il y a d'autres choses qu'on leur explique ... parce que aussi demander à une jeune fille de pratiquer la planification familiale c'est lui demander de faire le libertinage, des fois ça me dérange »(GD Mbour).

\section{DEMEDICALISATION}

L'étude montre que la plupart des personnes interrogées, aussi bien au niveau central que régional, connaissent beaucoup d'actions développées dans le cadre de la démédicalisation (Cf. Revue documentaire).

\section{Actions citées par les acteurs PF}

Ils font surtout référence aux avancées observées par rapport à la délégation des tâches. Ils font remarquer que concernant l'offre de services PF, c'est non seulement la technique qui a été démédicalisée, mais également l'information. Par exemple, l'offre de MLDA, notamment implants et DIU, par les ICP qui était jusque-là 
réservée aux médecins et sages-femmes. Certains acteurs ont aussi mentionné la mise en place en 2012 d'un plan de formation pour permettre aux matrones et ASC des cases de santé de mieux maitriser les techniques de communication par rapport au counseling, mais également pour pouvoir orienter vers les structures les mieux indiquées les femmes qui présenteraient des effets indésirables ou qui porteraient leur choix sur les MLDA. Cette formation avait également pour but de capaciter les matrones et ASC à l'OIP et à l'offre initiale de contraceptifs injectables sous forme intramusculaire (Depo Provera ${ }^{\circledR}$ ) et sous forme sous-cutanée (Sayana ${ }^{\circledR}$ Press). Certains acteurs indiquent que c'est suite aux résultats concluants de ces expériences que le MSAS a autorisé ces pratiques en 2014 sur la base d'une lettre circulaire. Une source indique que «Même s'il n'y a pas de loi formelle, le MSAS a fait des changements dans les PNP qui font référence à cela. Par exemple quand on dit que la case de santé est gérée par une matrone et que les produits PF doivent être disponibles au niveau de la case, cela suppose que la matrone peut les donner » (Partenaire technique).

Par ailleurs, certains acteurs informent d'initiatives ayant poussé un peu plus loin la démarche, à travers des études pilotes, en permettant l'offre de pilules et d'injectables par les relais communautaires en dehors des cases de santé.

"Maintenant, ily a des relais communautaires qui offrent la pilule et l'injectable. Donc là c'est un déclic que la PF a été transférée au niveau communautaire. Là aussi je pense que c'est une bonne chose. C'est une avancée. Nous avons démarré l'offre de pilules ... les relais communautaires ont maintenant élargi la gamme en offrant l'injectable depuis le mois d'avril [2015] et depuis ce mois on a plus d'une centaine de femmes qui sont déjà sous ces méthodes » (Représentant ONG).

"Ily a de réels progrès. Aujourd'bui, ily a beaucoup d'initiatives qui ont impliqué plusieurs acteurs qui ne sont pas spécifiquement du secteur de la santé. Il y a les relais, les matrones, les bajenu gox qui jouent un rôle dans la demande mais aussi la référence. Là, c'est vraiment une démédicalisation » (Représentant ONG).

"Ily a l'offre, il y a la sensibilisation. Il n'y a pas que le personnel médical qui offre. Il n'y a pas que le personnel médical qui fait la sensibilisation. C'est ça. Ce n'est pas seulement le personnel médical qui fait la sensibilisation dans les associations et autres... ily a des leaders qui sont dans les communautés à qui on parle. Il y a aussi les bajenu gox qui sont là, qui nous aident beaucoup »(Membre ECD Mbour).

Dans le cadre du développement de la PF communautaire, certains acteurs, notamment des représentants du gouvernement, signalent l'existence de la Politique de Santé Communautaire mise en place en 2013, et l'élaboration d'un Plan National Stratégique de Santé Communautaire en 2014 (Cf. Revue documentaire).

\section{Actions citées par les femmes bénéficiaires}

Les données recueillies dans le cadre des GD laissent penser que les communautés ont été informées des formations préalables reçues par les agents de santé du niveau communautaire pour l'offre de méthodes PF. Mais quasiment toutes les femmes savent que désormais elles peuvent bénéficier de l'offre de méthodes contraceptives par les matrones et les ASC au niveau des cases de santé.

"On les regroupe et les forme [les matrones et les ASC], on leur montre les différentes méthodes, et leur dit comment on les utilise une fois dans les villages » (GD Mbour).

"On a formé les ASC et les matrones à la PF c'est pourquoi ils ont commencé à en administrer [les méthodes]. En effet la demande en PF est tellement saturée que les sages-femmes et médecins ne peuvent plus tout faire »(GD Sandiara).

"Actuellement beaucoup de femmes se limitent au niveau des cases surtout celles qui habitent dans des zones éloignées...Mais celles qui habitent loin vont chez. la matrone ou chez. l'ASC pour qu'ils leur offrent le service. Tout le monde en dispose maintenant " (GD Fissel). 
"La formation, c'est bien ce qui a été fait pour permettre aux ASC et matrones d'offrir des services PF» (GD Sindia).

\section{Succès et réalisations de la démédicalisation}

Selon les acteurs PF, les succès et réalisations sont surtout visibles à travers l'élargissement de la gamme de méthodes au niveau des cases de santé, qui se limitait jusque-là à la pilule, au collier et au préservatif. En effet, la quasi-totalité des cases de santé, «97 pour cent» précise un représentant d'ONG, font l'OIP avec des matrones formées et en principe régulièrement supervisées. Ce même informateur précise que : "Pour le sous-cutané, on est à 100 pour cent de disponibilité au niveau des cases pour ... 4 régions. Mais, au niveau national ça fait 40 pour cent». Un représentant du Gouvernement va dans le même sens : "Actuellement il y a plus de 600 cases qui offrent linjectable (Depo IM) et 300 qui offrent le Sayana ${ }^{\circledR}$ Press à peu près. Donc avant ils ne pouvaient pas offrir ces méthodes, maintenant ils peuvent, en dehors de la pilule, du collier, du préservatif. Donc ça c'est des méthodes que les matrones, les ASC peuvent faire. Le counseling également est bien fait par ces matrones-là. Je pense que c'est des succès en terme de démédicalisation».

La prise en compte du volet communautaire par cette intégration de la PF dans les cases de santé a ainsi contribué à relever les indicateurs. Elle a aussi permis de rendre beaucoup plus accessibles les services.

"Pour nous le succès déjà c'est le fait que les matrones, quand elles sont formées et encadrées, qu'elles soient capables d'offrir le service, mais dans toutes les dimensions. C'est-à-dire non seulement elles accueillent la femme, elles lui font le counseling initial, elles offrent la méthode et surtout aussi elles reportent ...Pour moi, c'est important qu'on ait cette masse critique d'acteurs communautaires de santé qui soient capables d'offrir le service» (Représentant ONG).

"On a formé des relais communautaires, des ASBC [Agent de santé à base communautaire] d'un village. On les a formés sur l'offre initiale de pilule et ils sont retournés dans leur village. Ces relais ont commencé à faire le plaidoyer, des visites de proximité, ils ont sensibilisé, ils ont commencé à offrir la pilule au niveau communautaire. Ils sont partis en supervision et l'ICP était présent. L'ICP lui-même nous a dit que "ce que les relais communautaires ont offert en PF durant 2 mois je suis resté 7 ans sans l'offrir". Parce que les relais en l'espace de 2 mois ils ont pu enrôler, recruter et offrir presque 42 méthodes PF. Et lui pendant 7 ans il n'a même pas eu 10 cas. Donc cela veut dire que c'est un succès » (Représentant ONG).

"La démédicalisation a permis d'augmenter le nombre d'utilisatrices de la PF. Le niveau communautaire a une grande part dans les résultats que nous avons aujourd'bui, et que le personnel qualifié à lui tout seul ne pouvait pas avoir. Et donc tout cela contribue à la hausse du TPC. Même si l'offre est encore limitée, mais au moins elle est à proximité et les femmes ont plus de choix au niveau de la case de santé » (Partenaire technique).

Par rapport à la gestion de l'information, la mise en place d'un fichier communautaire PF au niveau de certaines infrastructures est une réalisation notée. De plus, certains répondants soulignent aussi l'élargissement de la panoplie des acteurs de l'offre avec, en dehors de l'intervention des matrones et des ASC, celle des relais communautaires dont la particularité est d'intégrer l'offre et la création de la demande. "Les relais, ils créent la demande et ils offrent en même temps. Les prestataires elles sont dans leur salle, elles ne peuvent pas créer la demande. On crée la demande, on leur réfere. Mais l'avantage du niveau communautaire, en même temps on crée la demande, en même temps on offre. C'est beaucoup plus bénéfique pour les clientes » (Représentant du Gouvernement). D’autres réalisations rapportées sont la réduction de la charge de travail du personnel qualifié ainsi que la contribution à la réduction des BNS. "Parce que la femme, peut-être même, elle peut avoir besoin de la planification familiale. Mais, elle n'a pas le temps d'aller sur 5 kilomètres, 10 kilomètres. Donc, le prestataire est tout à fait à côté. Ou bien, elle ne sait même pas où est ce qu'il faut aller la chercher. Donc c'est une stratégie conquérante, c'est une stratégie qui permet d'atteindre facilement les populations» (Représentant du Gouvernement).

Au-delà de la réduction du coût du service pour les populations, surtout rurales, avec la suppression des frais liés au transport, la démédicalisation permet aussi le maintien de certaines femmes dans le programme du fait de la proximité. 
"Ça réduit les déplacements...et ça réduit également les abandons parce que quand les services sont offerts, proches des domiciles, les gens sont plus à même de continuer parce que quelquefois il y a des abandons parce que les gens n'ont pas les moyens de continuer, d'aller aux rendez-vous » (Membre ECD Mbour).

"Ça réduit le coût de la prise en charge de ces dames. Parce qu'une dame qui devait payer le transport pour quitter sa localité et aller jusqu'à une autre localité, déjà c'est un coût. Pour les gens qui sont en milieu rural, qui ont des revenus qui sont assez. limités, le fait de réduire ce coût c'est déjà un grand succès » (Membre ECD Popenguine).

Pour les femmes bénéficiaires, les succès de la démédicalisation sont très visibles et se traduisent essentiellement par :

- La proximité/l'accessibilité géographique du service.

- Le gain de temps.

"Auparavant on venait à l'hôpital faire la queue, aller au marché et revenir encore faire la queue, rien que pour pouvoir faire le planning. De ce fait on perdait beaucoup de temps. Le repas n'était pas prêt à temps. Mais depuis on va chez. la matrone. Elle nous fait rapidement le planning 》 (GD Sindia).

- La confidentialité

"Je vois que la démédicalisation est une bonne chose pour la société car ily avait des contraintes. Parfois on veut se rendre à l'hôpital mais on a peur d'y rencontrer une connaissance. Tu connais la société sénégalaise. Tu peux discrètement la nuit te rendre chez la matrone et lui raconter discrètement ton problème sans que personne ne le sache »(GD Mbour).

- La disponibilité du service

«Elles sont plus disponibles. A n'importe quelle heure on peut aller les voir contrairement aux structures sanitaires où il y a des horaires à respecter. Puisque certains maris ne sont pas d'accord avec le planning on ne peut que nous rabattre sur les matrones et les $A S C »(G D$ Thiadiaye).

- L'amélioration de la santé des femmes rurales

- L'espacement des naissances

\section{Limites de la démédicalisation}

\section{Pour les acteurs PF :}

Pour eux, la plus grande faiblesse de la démédicalisation demeure l'absence de motivation des acteurs communautaires. De leur point de vue, bien que ces agents se soient engagés sur le principe du bénévolat, l'augmentation de leur charge de travail, due à leurs nouvelles compétences aurait pu entrainer des mesures d'accompagnement, mais ces mesures n'ont pas suivi sur le plan financier, ce qui ne facilite pas leur maintien dans le système.

"Ce qui n'a pas beaucoup marché c'est tout le côté financier parce que je vois qu'ily a des gens qui sont très impliqués mais qui ne sont pas payés, les matrones, les bajenu gox... Ils ne reçoivent pas une motivation suffisante, conséquente pour pouvoir continuer de travailler et j'ai peur qu'à la limite ils s'essoufflent parce que ça demande énormément d'efforts et c'est pas toujours facile d'entrer dans les maisons, de sensibiliser, parfois tu peux être mal accueilli, tu peux même subir des frustrations là-bas » (Représentant du Gouvernement).

"Jusqu'à présent il n'y a pas une motivation financière à l'endroit de ces agents communautaires-là, les ASC, les matrones, même les relais, les bajenu gox. On a beau parler de bénévolat mais en réalité nous pensons qu'il faut les accompagner sur ce plan-là. 
Donc c'est des problèmes qui sont tout le temps relatés pendant la supervision que nous faisons au niveau des cases 》 (Membre ECD Thiès).

Par ailleurs, beaucoup d'acteurs déplorent l'absence de législation pour encadrer l'offre de services par les acteurs du niveau communautaire. Les aspects législatifs posent d'autant plus de problèmes qu'ils constituent également un obstacle à l'offre potentiellement large de méthodes contraceptives par les pharmaciens.

"Les aspects de réglementation, ça, c'est aussi une limite. Il faudrait qu'on arrive, maintenant que ça se fait, à faire en sorte qu'il y ait une législation là-dessus. Jusque-là, on peut considérer que c'est un processus normal. Parce que le législateur, l'Etat, interviennent toujours lorsque la population s'est réellement appropriée de quelque chose » (Représentant du Gouvernement).

"Il y a des zones d'ombre. Les premiers textes réglementaires, les premiers textes en pharmacie, les premiers textes médicaux avaient délimité les acteurs et leurs responsabilités. Même le pharmacien n'avait pas le droit d'injecter, à plus forte raison l'ASC. Donc si aujourd'bui nous devons dire à cet ASC tu peux injecter le Sayana ${ }^{\circledR}$, tu peux injecter le Depo ${ }^{\circledR}$, il faut que les textes suivent. Même si les arrêtés du Ministère leur permettent de le faire, les textes ne les protègent pas. Sauf que cette loi sur la SR a des rubriques, des items qui peuvent les protéger donc il faut juste la faire appliquer et également mettre le plan d'action, ça veut dire le décret d'application... le communautaire n'est pas nouveau au Sénégal. Il faut juste les accompagner sur l'aspect juridique, réglementaire, législatif pour quand même les couvrir. On ne peut pas les mettre sur les activités alors que sur le plan juridique ils ne sont pas bien assistés» (Représentant ONG).

"L'autre aspect en matière de démédicalisation qui n'a pas marché c'est par rapport aux pharmaciens. On a autorisé l'injectable aux matrones alors que les pharmaciens sont des Docteurs en Pharmacie qui ont eu à faire des stages en soins infirmiers, qui ont validé les soins infirmiers, c'est-à-dire qui sont capables de faire des injections et pour lesquels cette méthode ne peut pas être donnée. Ils ne peuvent pas administrer parce que la loi ne leur permet pas d'offrir des services. C'est un peu paradoxal. Et si on voit le nombre de pharmacies et la répartition des pharmacies, on a une chance ou bien une potentialité d'angmenter le nombre de services qui offriraient des services de PF, tels que la pilule, mais l'injectable, même s'ils ne peuvent pas insérer des DIU ou bien des implants, mais au moins qu'on leur autorise pour l'injectable » (Représentant du Gouvernement).

L'absence de supervision régulière a été décriée par certains acteurs comme pouvant porter atteinte à la stratégie dans certaines zones dans la mesure où ces nouvelles compétences nécessitent un suivi rapproché, particulièrement l'offre d'injectables qui reste un acte médical.

"Là où ça marche moins, c'est le fait que les districts s'approprient les interventions et fassent un suivi régulier. Ils font des supervisions, ils font des stratégies avancées. Mais, j'avoue que c'est un peu ça le défi, la régularité des supervisions et de l'encadrement par les prestataires. Parce que nous n'avons pas la vocation de remplacer le Ministère, nous appuyons juste le Ministère. Et donc, ce sont les professionnels de la santé qui font les formations, qui sont censés aussi organiser, et suivre l'encadrement. »(Représentant ONG).

"Ces communautaires-là ce ne sont pas des agents de santé et on leur confie des tâches de santé donc il faut un suivi régulier et une supervision régulière pour éviter les problèmes. C'est ça qui manque un peu, parce que un seul cas d'échec ça peut gâcher beaucoup de choses. » (Membre ECD Thiès).

En outre, quelques acteurs notamment au niveau central, affirment avoir constaté une lenteur, voire des hésitations de l'Etat dans la mise en place des expériences innovantes ainsi que dans leur passage à l'échelle, ce qui constitue l’un des principaux freins à la démédicalisation.

"On a tardé dans cela. Il y a eu des retards dans l'application des expériences innovantes. Depuis 2009 déjà, dans les réunions on parlait de l'introduction des injectables, et c'est seulement là en 2015 que ça a été introduit » (Partenaire technique). 
"Depuis 2012, c'est seulement en 2015 qu'ils ont écrit une note pour accepter la phase test. On a perdu 3 ans.... C'est un peu la lenteur dans l'acceptation. Actuellement on est en phase test. ... mais c'est circonscrit, on attend que ce soit concluant pour qu'on puisse mettre à l'échelle » (Représentant ONG).

Concernant les outils de gestion, même si de nombreux acteurs déclarent que les agents de santé communautaire arrivent à faire assez correctement leurs rapports d'activités, ils reconnaissent aussi que c'est parce que ces derniers se font souvent aider par des proches ou des agents de développement communautaire (ADC), hormis quelques rares exceptions (cas des ASC qui ont généralement un niveau d'instruction un peu plus élevé). Ils sont d'avis que le niveau d'éducation, très bas de la majorité de ces agents (particulièrement chez les matrones et les relais), pose problème pour le rapportage de leurs activités. «Le niveau d'étude des relais sonvent c'est un niveau très bas. Et souvent les outils de collecte sont un peu à un niveau élevé et les relais n'ont pas souvent la capacité à pouvoir bien renseigner les outils de collecte » (Représentant ONG).

La gestion des effets secondaires par les agents de santé communautaire apparait pour quelques acteurs comme un frein à la démédicalisation : "Ça ne peut pas marcher, ce sont des gens qui peuvent reconnaitre les effets secondaires, mais qui n'ont pas la technicité de prendre en charge tous les effets secondaires. Donc elles sont obligées de référen» (Membre ECD Mbour).

Aussi, le manque d'intégration systématique des cases de santé dans le circuit d'approvisionnement participe à maintenir les ruptures de stocks à ce niveau de la pyramide, d'où la nécessité d'adresser cette question. "Le circuit actuel se limite seulement au niveau poste, pas à la case. Alors les cases quelquefois elles connaissent quelques ruptures, de temps en temps il y a un petit dysfonctionnement» (Représentant ONG). Certains notent d'ailleurs l'insuffisance de cases de santé : "On a 2900 cases au Sénégal pour 19000 villages donc ça veut dire qu'il n'y a environ que un tiers des villages qui sont couverts. Donc ça veut dire qu'il y a beaucoup de villages qui n'ont pas de cases 》(Représentant du Gouvernement). D’autres font remarquer que certaines femmes préfèrent délaisser la case au profit du poste de santé. Ils l'expliquent par deux (2) principales raisons : soit elles se cachent, à l'instar de nombreuses femmes rurales, soit elles n'ont pas confiance.

"Ce qui n'a pas très bien marché c'est d'abord la fréquentation des cases de santé. Aujourd'hui si nous avions à calculer l'offre par rapport aux cases de santé, comparé à l'offre dans les structures comme les centres et les postes de santé, vraiment c'est pas comparable. On a l'impression qu'ily a une fréquentation certes dans les cases par rapport à la PF mais les taux de fréquentation sont encore faibles. On peut voir une case qui, par exemple, durant un mois ne reçoit que 2 ou 3 clientes. Mais l'explication peut être diverse et variée » (Membre ECD Thiès).

"C'est un problème de confiance aussi. Parce que nous savons tous que ce sont des agents qui sont issus de la communauté, qui sont formés dans le tas d'une certaine manière. Et c'est pas toujours évident qu'au retour dans leur communauté qu'on leur fasse confiance. Ca aussi c'est des réalités qui sont là» (Membre ECD Thiès).

Une source indique que la situation peut également être liée à l'insuffisance des services offerts au niveau de la case. "Aujourd'bui nous avons l'impression que les populations, ils ont des besoins qui dépassent un peu ce que la case leur offre. Il y a déjà un problème de disponibilité ou de satisfaction des besoins par rapport à l'offre qui existe dans la case. Donc les populations sont obligées parfois de venir jusqu'au niveau du poste de santé pour pouvoir satisfaire leurs besoins. Donc c'est par rapport à l'offre qui n'est pas toujours adéquate par rapport à la demande des populations » (Membre ECD Thiès).

\section{Pour les femmes bénéficiaires :}

Le fait d'avoir permis aux agents de santé communautaire d'offrir la PF ne rassure pas certaines femmes, malgré tous les avantages que cela représente pour d'autres. En effet, leur manque de confiance est manifeste et pourrait s'expliquer essentiellement par le faible niveau d'éducation de ces agents. 
"Moi ce qui me rassure c'est d'aller dans les postes de santé pour faire le planning. C'est ce qui me rassure et c'est ce que je fais....aller dans les postes de santé où elles ont étudié et appris ça. J'ai plus d'espoir en eux qu'aux matrones et ASC » (GD Mbour).

L'insuffisance de cases de santé au niveau communautaire apparait également comme un frein à la démédicalisation de l'avis de quelques femmes, à l'instar de celle-ci qui affirme que "Ce qui ne marche pas c'est que beaucoup de villages n'ont pas de case de santé; si on avait construit des cases de santé dans tous les villages du Sénégal ça marcherait mieux. Ainsi elles sont obligées de se déplacer pour trouver des cases de santé et c'est fréquent, même les villageoises aux alentours de Mbour le font. Donc pour que ça marche mieux il faut des cases de santé partout» (GD Mbour).

\section{Opinions sur l'offre de MLDA par les agents de santé communautaire}

Parmi les acteurs PF, les avis sont partagés sur l'idée de permettre ou non aux ASC et matrones d'offrir des MLDA. En effet, même si quelques personnes interrogées pensent que les agents communautaires sont en mesure de le faire, et qu'on devrait le leur permettre, la majorité pense qu'on devrait avancer prudemment. L'approbation concernerait spécifiquement les implants, dont la pose demande moins de technicité médicale que le DIU, et l'anneau vaginal qui devrait bientôt rejoindre la gamme.

Les acteurs qui sont favorables à l'offre de MLDA par les agents de santé communautaire justifient leur position par le fait que cela permettrait de booster les indicateurs, qu'il y a une facilité d'utilisation des nouveaux types d'implants, et qu'il s'agit d'une expérience déjà en cours dans d'autres pays. Ils précisent tout de même qu'il faudrait que ces agents soient bien encadrés par rapport à la formation, au suivi et à la supervision, et qu'ils soient motivés.

"Au moins l'implant. Surtout qu'il y a des types d'implants maintenant qui sont assez. spécifiques, avec le Jadelle ${ }^{\circledR}$ et l'Implanon ${ }^{\circledR}$. Si ça arrive, ça devrait pouvoir aider facilement. On a parlé de l'anneau vaginal. Il n'y a pas de problèmes. Donc voilà, toutes ces possibilités sont là. Encore il y a une large perspective pour avancer sur ces questions » (Représentant du Gouvernement).

"Je ne sais pas si pour le DIU ils pourront le faire, mais je pense que pour l'implant ça peut se faire. Et je pense qu'il y a même des pays où on a déjà commencé à tester cela et ça se passe bien. Donc il faut y aller étape par étape. Mais il faut apprendre des bonnes pratiques qu'il y a par ailleurs, on n'a pas besoin de réinventer la roue ou de réfléchir pendant 10 ans sur une question. Il suffit juste de regarder ce qui se fait par ailleurs, pourquoi ça marche, comment ça marche, d'étudier et de l'adapter. Ca s'appelle du benchmarking tout simplement » (Représentant ONG).

"Je ne dis jamais non ou bien je ne dis jamais jamais...l'acteur communautaire, s'il est formé, s'il est encadré, je dis bien s'il est bien suivi, s'il a tout ce qu'il faut, cet acteur communautaire est aussi capable. Peut-être que la théorie va faire la différence entre l'acteur communautaire.... Actuellement je suis plutôt communautaire, je sais de quoi est capable l'acteur communautaire, je sais de quoi il n'est pas capable. Un acteur communautaire qui a utilisé le misoprostol, qui gère le misoprostol... le misoprostol n'est pas fourni au niveau du centre de santé. S'il lui est permis de faire ça, je pense que le reste ne sera pas diable 》 (Membre ECD Thiadiaye).

En revanche, ceux qui sont opposés à l'idée de permettre aux agents communautaires d'offrir les MLDA, essentiellement des prestataires, avancent des arguments liés à la complexité de l'acte compte tenu de la faible compétence de ces agents en la matière, du manque de matériel ou du respect des règles d'asepsie. Ils citent aussi parfois simplement la jalousie.

"On est jaloux de ce qu'on a appris pendant des années... Je suis jalouse. Oui, même pour les injections. J'ai été frileuse. ... Pourtant elles sont là, elles offrent, et c'est passé, la pilule a été amère mais quand même elle est passée. Et pourtant ça passe. Il faut combien d'années pour former une sage-femme, il faut combien d'années pour que la sage-femme sache comment faire une 
injection intramusculaire. C'est pourquoi je suis jalouse. Et il faut combien de temps pour former une matrone ou bien un ASC, et on délègue. ... Je vais l'accepter, je l'accepterai au fur et à mesure que les années passent, mais dans un premier temps ce n'était pas facile » (Membre ECD Thiès).

"Les implants, ils ne peuvent pas. C'est un peu chirurgical et c'est un peu difficile. Peut-être à la longue... je ne sais pas... même les sages-femmes qui ont fait la formation, il leur faut une formation complémentaire pour qu'elles puissent faire correctement les implants. Le DIU, on n'en parle même pas. Il y a aussi qu'il faut stériliser le matériel et ils n'ont pas le matériel adéquat pour stériliser» (Membre ECD Mbour).

"Je pense que c'est ça qu'ils peuvent offrir pour le moment parce que concernant les méthodes longue durée, non seulement il y a la compétence, il y a également un équipement qu'il faut et je pense que ce qu'on a délégué déjà c'est bon, il faut un peu de recul, qu'on évalue, qu'on voit les avantages, les inconvénients et autres avant de songer à leur déléguer autre chose» (Membre ECD Mbour).

Pour les femmes bénéficiaires, quasiment toutes les participantes sont opposées à l'offre de MLDA par les agents de santé communautaire. Elles justifient cette opposition par la complexité de ces méthodes qui nécessitent certaines compétences qu'ils n’ont pas, mais aussi par l'absence du plateau technique que cela exige.

"Ils peuvent faire le Depo®, les pilules. Ils ne peuvent pas offrir les autres méthodes car pour ces méthodes il faut un personnel de santé expérimenté et qualifié. Par erreur il peut arriver qu'ils le placent là où il ne faut pas de même que l'appareil [DIU]. C'est pourquoi ils ne peuvent pas offrir les autres méthodes pour le moment donc ils doivent se limiter aux pilules et à l'injectable 》(GD Mbour).

«Parce qu'ily a des instruments que les matrones et ASC ne disposent pas encore pour offrir les autres méthodes» (GD Sandiara).

"Chacune sa spécialité et sa compétence. On ne peut pas tout faire. Aux matrones ce qu'elles maitrisent et peuvent faire et laisser les sages-femmes aussi faire ce qui relève de leurs compétences pour éviter toute complication. La santé est sensible et capitale. Tout ce que l'on doit faire ou mettre sur le corps bumain doit être clair, sûr et rassurant »(GD Popenguine).

\section{DECENTRALISATION}

\section{Actions citées par les acteurs PF}

La plupart des acteurs PF interrogés connaissent de nombreuses actions menées dans le cadre de la décentralisation. Par exemple, les changements opérés dans les PNP en vue d'intégrer la disponibilité des produits PF au niveau le plus bas de la pyramide sanitaire ont été soulignés. Ces dispositions matérialisent la décentralisation de l'offre de PF. En dehors des structures sanitaires, les stratégies avancées (ex : cliniques et équipes mobiles) au profit des femmes résidant dans les zones les plus reculées ont aussi beaucoup contribué à cette décentralisation.

"Vous parcourez le Sénégal, partout il y a des tableaux et des affiches qui montrent que c'est des services de PF, donc c'est allé même jusqu'au niveau cases de santé, avant c'était juste au niveau des postes de santé mais maintenant au niveau des cases on peut accéder à la PF » (Représentant ONG).

De même sur le plan technique, non seulement la prise de décisions n'est plus centralisée, comme le souligne ce représentant du gouvernement : "Ce n'est plus une histoire de savants, une histoire d'experts, une bistoire où tout se décide au niveau central », mais les formations se font aussi in situ. Dans ce cadre, l'initiative du Tutorat Plus a permis de fournir une formation professionnelle aux prestataires de soins.

Pour la majorité des acteurs PF, l'IPM est considéré comme l'action la plus innovante et la plus déterminante de décentralisation des produits PF grâce au système de dépôt-vente (paiement après-vente) dont ne bénéficient pas les autres produits essentiels. Cette initiative, mise en place dans un souci d'équité en réponse aux problèmes 
de ruptures de stocks, a fortement facilité la distribution des produits pour les rendre disponibles à tous les niveaux.

"Ily a la formation du personnel qui se fait à tous les niveaux. Ce qui fait qu'actuellement, l'offre de services PF est disponible à tous les niveaux de la pyramide sanitaire, même jusque dans les cases de santé. L'approvisionnement des PPS [Point de Prestation de Services] en produits a été également décentralisé. Aujourd'hui grâce au Push Model, on note une disponibilité des produits même dans les structures les plus périphériques telles que les cases de santé » (Acteur PF Thiès).

En termes d'offre, au-delà de la méthode, l'information a aussi été décentralisée à travers l'implication des acteurs et des radios communautaires pour faciliter le choix éclairé des femmes. Par ailleurs, certaines sources évoquent la stratégie des sages-femmes itinérantes (SAFI) qui améliore également l'offre de services SR en général et de PF en particulier (Cf. Revue documentaire).

Dans le cadre du transfert des compétences de l'Etat aux instances décentralisées, certaines collectivités locales commencent à s'investir dans la construction de structures sanitaires, le financement de la PF, et le recrutement de personnel qualifié, notamment les sages-femmes.

"On a eu des cas dans la région de Kaolack, on a été dans un des villages. Le maire, il est très jeune. Il a à peine 30 ans et il a eu une bonne initiative. Dès qu'il est arrivé, il a recruté à travers la mairie, une sage-femme qui est là-bas, et qui est bien intégrée dans la population » (Représentant ONG).

"Aussi, les élus locaux, des comités de santé ont suivi dont certains maires qui ont procédé au recrutement de personnel et de mise à disposition de fonds pour les produits SR» (Représentant ONG).

"Ily a également l'implication des maires dans la région de Dakar, avec l'appui du Réseau Siggil Jigeen... Hier, il y avait une activité avec tous les maires de Pikine, Guédiawaye... qui se sont engagés vraiment à augmenter leur ligne budgétaire pour appuyer la PF » (Représentant du Gouvernement).

"Aujourd'hui nous voyons des maires qui sortent des lignes budgétaires pour acheter des produits PF. Je l'ai vu à Pikine Ouest, je l'ai vu à Djidah Thiaroye Kao, je l'ai vu à Guédiawaye, des maires qui achètent des produits et le mettent à la disposition de leurs postes. Ça c'est un engagement parce qu'au début tout ce qui a été comme portefenille budgétaire, comme fonds de dotation, la PF n'était pas sentie, donc si aujourd'bui les maires se sentent vraiment intéressés jusqu'à acheter des produits, jusqu'à financer des stratégies avancées, appuyer les ONG dans les stratégies avancées, dans ce qu'on appelle les journées des communes avec la PF c'est déjà quelque chose » (Représentant ONG).

\section{Actions citées par les femmes bénéficiaires}

Les femmes bénéficiaires ont surtout fait référence à la possibilité depuis ces dernières années de pouvoir bénéficier de l'offre de services à tous les niveaux, et aussi bien en milieu urbain qu'en milieu rural.

"Le gouvernement a permis aux femmes de pouvoir faire la PF partout où elles vont, pour leur faciliter la tâche, pour leur éviter le déplacement» (GD Fissel).

"Toutes les femmes ont accès à la PF avec la décentralisation. On n'a plus besoin d'aller dans les grandes villes pour seulement faire le planning 》(GD Mbour).

"Quel que soit l'éloignement d'un village, on a fait en sorte qu'il y ait une case de santé pourpermettre à tout le monde d'y accéder [à la PF] »(GD Thiadiaye). 


\section{Succès et réalisations de la décentralisation}

\section{Pour les acteurs PF}

Du point de vue des acteurs, les succès sont nombreux aussi bien en milieu urbain qu'en milieu rural. Ils ont notamment cité l'augmentation du TPC, l'amélioration de l'accessibilité géographique et la réduction du coût des services. En milieu rural, cela est dû en particulier à l'offre de services au niveau communautaire (cases de santé, quartiers et villages) et au renforcement des capacités des prestataires et des acteurs locaux. En outre, les acteurs ont souligné la disponibilité et la diversité des méthodes contraceptives, ce qui permet d'offrir un plus large choix aux femmes.

"Avant on disait que dans la supervision "il faut voir s'il y a au moins trois méthodes contraceptives modernes". Je pense qu'on n'entend plus parler de ça. Maintenant, on est allé un peu plus loin que ça. Ça aussi, je crois que c'est un succès. » (Représentant du Gouvernement).

Certaines sources indiquent aussi le fait que dans la mise en œuvre du PANPF, loin de l'approche «top down » précédemment appliquée, il y a une réelle implication du niveau communautaire et surtout une appropriation par les régions de l'atteinte des objectifs. Cette implication se traduit par l'engagement des Médecins Chefs de Région (MCR) à revoir à la hausse les objectifs régionaux et à les atteindre, et par l'élaboration d'un plan d'action régional, sur la base duquel les structures de santé réalisent depuis 2012 leur plan d'action PF.

"Je pense que pour la définition des objectifs, ce sont les Médecins Chefs de Région qui ont donné leurs objectifs, rien ne leur a été imposé. Chacun en fonction de ses potentialités, en fonction des besoins non satisfaits de sa région, des potentialités des femmes en âge de reproduction dans sa région, chacun s'est dit voici ce que je peux contribuer pour l'atteinte de l'objectif 》 (Représentant du Gouvernement).

"Bien que la PF soit quelque chose de basée sur le choix libre et éclairé, les différents responsables sanitaires se sont appropriés et ont essayé de faire comprendre aux prestataires l'intérêt de s'impliquer dans ce plan » (Représentant du Gouvernement).

La responsabilisation du niveau communautaire semble être pour beaucoup une réalité, et ils signalent qu'elle est très visible à travers l'exercice de nouvelles prérogatives et l'engouement dans la mise en œuvre du PANPF. «Nous avons tenu la revue à mi-parcours du plan et on a senti une certaine émulation positive entre les régions médicales, mais également ça s'est traduit au niveau des districts... Il faut vous rendre dans les réunions de suivi de nos plans au niveau régional, vous vous rendrez. compte que c'est comme si ily avait une saine compétition entre les districts et je pense que c'est quelque chose qui est extraordinaire. Les gens maintenant rivalisent d'ardeur sur les bonnes pratiques, sur le recrutement des femmes, l'offre de services est améliorée, ily a plus d'adhésion, on sent un engouement autour de ce plan et ça je pense que c'est quelque chose qui est excellent » (Représentant du Gouvernement).

En outre, certains acteurs pensent qu'il y a une meilleure emprise de cette politique de PF par le niveau opérationnel. "Il suffit de vous rendre sur le terrain pour voir comment les gens maitrisent les concepts, les différents domaines stratégiques et dans chaque domaine ce qu'ils sont en train de faire en termes de formation, en termes de création de la demande, en termes de mobilisation sociale, en termes d'émissions radio, en termes de disponibilité, de plaidoyer. Je pense que ça c'est un succès» (Représentant du Gouvernement).

Certains acteurs soulignent que cette décentralisation a permis de rendre l'offre de services PF équitable par l'augmentation du nombre de PPS et de valoriser le niveau communautaire. Comme l'indique cette Autorité sanitaire au niveau local à propos des nouvelles prérogatives des agents : «ils ne sont pas limités à la routine, à faire des pansements, à distribuer des comprimés». 


\section{Pour les femmes bénéficiaires}

Les plus grands succès de la décentralisation résident dans l'accessibilité géographique à la $\mathrm{PF}$, la réduction du coût du service grâce à sa proximité, et le gain de temps engendré. En outre, certaines femmes ont le sentiment que l'offre de service est devenue plus équitable.

"Maintenant c'est plus proche et tu marches, tu vas dans la structure de santé, on t'offre la méthode et tu rentres chez. toi, c'est plus reposant, tu n'es pas obligée de voyager, d'aller et de revenir » (GD Fissel).

"Il y a des avantages pour nous parce qu'actuellement quel que soit le lieu où tu te trouves tu peux le faire là-bas. C'est reposant pour les femmes. Au lieu de payer le transport pour aller loin, tu le fais dans ta localité »(GD Thiadiaye).

"On ne peut plus faire de différence entre les villageoises et les citadines. Tout le monde est sur le même pied d'égalité, parce que la PF est partout, les femmes ont maintenant du temps pour elles-mêmes et pour s'occuper de leur mari 》(GD Mbour).

\section{Limites de la décentralisation}

Alors que les femmes bénéficiaires considèrent que dans la décentralisation tout a bien marché, pour les acteurs PF, certains aspects pourraient être améliorés.

En ce qui concerne le financement de la PF, au-delà des efforts constatés et des dispositions prises sur le plan budgétaire, certains acteurs notent une insuffisance dans la participation des collectivités locales sur le plan opérationnel. En effet, elles dépendraient encore beaucoup trop de l'Etat concernant, par exemple, la prise en charge de leur personnel. Cela s'expliquerait par un manque de moyens, mais surtout par un manque d'appropriation de la prise en charge de la PF.

"Pour la décentralisation, ce que je constate, c'est qu'on ne sent pas trop l'implication des collectivités locales dans le financement de la PF alors que c'est quelque chose qu'elles sont en mesure de faire. Actuellement dans le budget de ces collectivités, l'Etat a prévu quelque chose pour le secteur de la santé, mais dans la pratique, c'est quelque chose que l'on ne sent pas » (Acteur PF Thiès).

"Tant qu'ils n'ont pas leur propre personnel, tant que le personnel continue de dépendre de l'Etat du Sénégal, ily a quelque chose qui va grincer quelque part» (Représentant $\mathrm{ONG}$ ).

"Il ne sert à rien de décentraliser si les moyens ne suivent pas. La santé est une compétence transférée, c'est décentralisé donc cela veut dire que les collectivités locales doivent prendre en charge la PF mais ce n'est pas encore fait.... à part récemment où j'ai entendu qu'ily a une collectivité locale à Dakar qui a inscrit 10 millions, mais ça ne bouge pas »(Représentant ONG).

De même, l'insuffisance de structures de santé, notamment de cases de santé, limite la couverture des interventions et pose un frein à la décentralisation. Des gaps ont également été observés pour les stratégies avancées.

Pour le système d'information, les compétences nécessaires pour la gestion du fichier PF poserait problème. Aussi, le mode de recrutement du personnel qualifié, notamment les sages-femmes, souvent basé sur la contractualisation, constitue une difficulté pour leur maintien dans les programmes.

"Le seul problème évoqué c'est que les comités payent pendant des années, après si ces gens-là sont recrutés au niveau de la fonction publique ou bien bénéficient d'une contractualisation au niveau du Ministère, on les prend, on les met ailleurs, après ça pose problème. Après c'est un éternel recommencement, on est à la recherche d'autres gens, on va ensuite essayer de contractualiser avec d'autres sages-femmes pour reprendre encore la formation. C'est ce qui est dommage» (Membre ECD Popenguine). 
L'offre limitée de méthodes au niveau des cases de santé, notamment l'absence des MLDA, est considérée par certains acteurs comme une limite de la décentralisation.

"Ca veut dire qu'aujourd'bui il y a des produits, si les populations les plus reculées veulent adbérer à ces produits-là, elles sont obligées encore de faire des kilomètres. L'offre est encore limitée » (Membre ECD Thiès).

A en croire certains, des irrégularités sont encore constatées dans le suivi et la supervision, qui constituent des exigences dans le cadre du transfert de compétences.

"Quand on décentralise aussi, cela ne signifie pas négligence. Tu ne t’adosses pas sur l'individu et que tu l'oublies. Ce que nous avons remarqué d'une façon générale, c'est que le suivi, la supervision n'était pas au rendez-vous. Ça quand même ça reste » (Membre ECD Thiès).

\section{VUE GLOBALE DE L'APPROCHE 3D}

L'étude montre que la mise en œuvre de l'approche 3D n'est pas perçue de la même manière par les personnes interrogées. Les avis sont partagés sur le « $\mathrm{D}$ » qui a le plus progressé durant ces cinq (5) dernières années.

Selon la majorité des acteurs PF du niveau central, ce sont les actions accomplies dans le cadre de la «Démocratisation » qui seraient les plus visibles du fait des réalisations observées sur le plan politique et programmatique, des résultats des campagnes de communication, et de l'implication d'autres acteurs : société civile, secteur privé (commercial et à but non lucratif) et autres ministères. De leur point de vue, « Démédicalisation » et « Décentralisation » seraient freinées par la déficience des infrastructures sanitaires, la pénurie du personnel qualifié, l'insuffisance de motivations financières, et la réticence de certains prestataires.

Par contre, pour la majorité des acteurs PF du niveau opérationnel, c'est la « Décentralisation » qui aurait le mieux progressé. Pour eux, ses réalisations sont plus tangibles grâce au rapprochement des services des populations, au renforcement des capacités des prestataires et à la disponibilité des produits. La "Démocratisation » serait encore limitée, notamment l'accès aux services dans certaines zones, et la «Démédicalisation » par le manque de motivation du personnel communautaire.

"S 'ily a des efforts à faire c'est par rapport à la démocratisation. Parce qu'aujourd'bui ce serait bien que les populations qui vivent en milieu reculé aient les mêmes chances que les populations qui vivent en milieu urbain. Ce sont des citoyens sénégalais. C'est ça la démocratie » (Membre ECD Thiès).

"Pour la Démédicalisation ce qui manque un peu ce sont les mesures d'accompagnement à travers une supervision régulière et une bonne motivation des agents de santé communautaire » (Acteur PF Thiès).

Les femmes bénéficiaires pensent également en majorité que la «Décentralisation » fait partie des réalisations les plus importantes des dernières années.

Mais au-delà de ces divergences d'opinions, acteurs PF et femmes bénéficiaires s'accordent à dire que les 3D sont complémentaires, interdépendants, et que l'un ne peut aller sans l'autre. Comme le dit ce parlementaire : «La démocratisation va de pair avec la décentralisation. Pour pouvoir démocratiser, on fait dans un système décentralisé. Parce que ça donne l'accès à tous les niveaux de la pyramide sanitaire. Donc, on ne peut pas démocratiser sans ce support décentralisé. Et c'est parce que ça, ça existe qu'on a la possibilité de démédicaliser. Les mathématiciens vous parleront de transitivité».

De plus, l'approche 3D est perçue comme une bonne approche du fait, entre autres, de la dimension multisectorielle qu'elle intègre. Il s'agit d'une approche innovante qui gagnerait à être systématisée et mieux vulgarisée pour une bonne appropriation des parties prenantes et comme l'indique un partenaire technique : 
«c'est une approche innovante au point que certains pays de la sous-région comme le Togo commencent à se l'approprier». Donc cette approche doit être démocratisée davantage.

«C'est une très bonne approche qu'il va falloir systématiser, réellement. V raiment systématiser réellement, mieux l'apprendre aux gens, mieux la vulgariser au niveau central, au niveau régional, au niveau départemental, à tous les niveaux. Et que les gens comprennent de quoi il s'agit, que les gens s'engagent avec des lignes directrices vers justement une bonne application de cette approche » (Représentant ONG).

"C'est une bonne approche, c'est une bonne stratégie, qui demande juste à ce que tout le monde soit vraiment, vraiment informé, au courant, de tout ce qu'ily a dans cette approche 3D, parce que je suis persuadée qu'il y a beaucoup de personnes qui ont beaucoup d'idées sur comment est-ce qu'on doit mener cette approche 3D » (Représentant ONG).

De l'avis de la majorité des acteurs interrogés, notamment du niveau régional, la communication autour de ce concept est d'autant plus importante qu'ils la considèrent comme nouvelle sur le plan conceptuel certes, mais pas sur le plan opérationnel. La réalisation de cette étude a été pour eux l'occasion de se rendre compte qu'il s'agit d'une approche qu'ils appliquent quotidiennement dans le cadre de leurs activités, sans même s'en rendre compte.

«En fin de compte, l'approche 3D, c'est quelque chose que nous faisons chaque jour sans même le savoir. Donc cette étude est vraiment une bonne initiative. Il suffit juste de la vulgariser [l'approche] pour que les interventions soient mieux structurées et que les acteurs puissent mettre un cadre à ce qu'ils font » (Acteur PF Thiès).

"Là, ça devient beaucoup plus concret après cette discussion, mais c'était quelque chose de très abstrait. Peut-être que les gens le font déjà même, mais ils ne savent pas que ça s'intègre dans une certaine approche. Et je pense que la communication à ce niveaulà, auprès des personnes concernées, des personnes clés ressources, ce serait une très bonne chose. D'autres idées peuvent sortir, qui pourront alimenter cela » (Représentant du Gouvernement).

"Je pense que c'est une excellente étude parce que ça nous permet même de revoir les choses, mieux nous impliquer et de mieux mesurer l'impact de ce que nous faisons parce que ce n'était pas évident pour les 3D....c'est quelque chose que nous faisons réellement, mais ça, ça nous permet de systématiser et de mettre dans des tiroirs chaque activité que nous menons parce que ce sont des choses que nous faisons mais très sincèrement là je suis séduite par cette approche-là....an départ j’étais un peu sceptique, je me disais "qu'est-ce que je vais dire à propos de cette approche que je ne maîtrise pas", mais après je me suis dit "tiens, c'est vraiment ce que nous faisons" mais on mène des activités sans pouvoir les mettre dans des tiroirs et c'est une excellente approche » (Membre ECD Mbour).

Les acteurs, aussi bien au niveau central que régional, sont tellement séduits par cette approche des 3D et par ses résultats, qu'ils pensent à l'unanimité qu'elle devrait être reconduite pour servir de ligne directrice à l'élaboration du prochain PANPF. Cependant, ils soulignent comme préalable, l'évaluation du plan en cours pour voir son impact réel.

«On a vu les résultats, je pense qu'on ne change pas une stratégie qui gagne 》 (Membre ECD Thiès).

"Je pense que c'est quelque chose que l'on peut reconduire pour le prochain plan. Mais il faut d'abord l'évaluer. Mais c'est une très bonne approche » (Partenaire technique).

Par rapport à la mise en œuvre de l'approche 3D à travers le pays, la plupart des acteurs pense qu'elle est mieux développée dans les régions qui bénéficient de la présence de nombreux partenaires (ex : Dakar, Saint-Louis, Thiès). Pour les régions ayant connu des freins, en dehors de l'absence ou de la rareté de partenaires, d'autres facteurs ont été évoqués tels que les pesanteurs socioculturelles, le refus des prestataires d'exercer dans ces zones, ou le manque de dynamisme et d'appropriation du programme par les MCR. 


\section{EFFETS PERÇUS DE L'APPROCHE 3D}

Selon les acteurs PF, plusieurs effets peuvent être attribués à la mise en œuvre de l'approche 3D, notamment: l'accessibilité des services PF aux populations, la fréquentation accrue des structures de santé, la hausse du TPC, le renforcement des capacités des acteurs communautaires, la diminution des grossesses à risque, une meilleure acceptation/adhésion à la $\mathrm{PF}$, la disponibilité des produits, et la baisse de la mortalité maternelle et infantile.

"Pour moi l'amélioration de la prévalence contraceptive dont on parle, c'est par rapport à ça. C'est par rapport à tous les efforts qui ont été faits pour améliorer l'accès aux services, améliorer l'accès aux médicaments, réduire un peu les barrières socioculturelles. Donc, je me dis que cette approche-là y est pour quelque chose, même si les gens peuvent faire l'approche sans savoir qu'ils le font " (Représentant ONG).

"Cette approche a beaucoup contribué au relèvement du taux de prévalence contraceptive. Cependant il faudra une évaluation plus rigoureuse pour évaluer son impact réel. Du côté de la demande, cette approche a permis de lever certaines barrières socio-culturelles. Ily a plus de religieux qui sont maintenant favorables à la PF. » (Représentant ONG).

"Les indicateurs parlent d'eux-mêmes. Et donc on voit réellement quel est l'impact de la décentralisation. Parce que les indicateurs sont en train de remonter vraiment dans les différentes régions, ce qui fait qu'on a pu avoir au niveau central 12 pour cent, 16 pour cent et maintenant 20 pour cent. Et on voit que ce n'est pas Dakar qui fait ce grand bond....mais il y a vraiment une performance des régions réputées difficiles où on ne s'y attendait pas. Ce sont quand même des choses qu'il faut saluer, qu'il faut mettre en exergue » (Représentant ONG).

Pour avoir des résultats encore meilleurs, ces acteurs préconisent la mise à l'échelle de la délégation des tâches, le renforcement des stratégies avancées et mobiles, l'implication des leaders communautaires, et l'information des acteurs sur l'approche.

"Il faut qu'ily ait une mise à niveau ou bien une orientation, information des gens sur l'approche. Il faut que cet outil-là, soit beaucoup plus systématiquement utilisé dans la mise en cuvre des interventions »(Représentant ONG).

"Il y a une meilleure adhésion des hommes, une meilleure adbésion des femmes et une meilleure adhésion des chefs religieux. Je pense que le Sénégal est en train de démontrer qu'avec une bonne implication de tous les acteurs on peut doubler son TPC en 3 ans » (Représentant du Gouvernement).

Comme l'affirme un acteur : «il est possible de faire des bonds très significatifs si toutes les politiques sont réunies». Mais certains acteurs, notamment du niveau central, d'attirer l'attention sur la nécessité de renforcer la coordination et la multisectorialité qui ne leur semble pas tout à fait effective.

"Pour que ces résultats soient meilleurs, il faut que tout le monde se sente concerné. Et ainsi il y aura de meilleures ressources (multisectorialité). Si l'aspect multisectoriel marche, on aura de meilleurs effets. »(Partenaire technique).

Les femmes bénéficiaires apprécient aujourd'hui les effets de l'utilisation de la PF, tels que l'espacement des naissances, la baisse de la mortalité maternelle, et le maintien des filles à l'école. Cela est dû, selon elles, aux résultats des actions menées dans le cadre des 3D, tels que l'accessibilité géographique et financière aux méthodes contraceptives, l'amélioration des connaissances des populations sur la PF, la disponibilité des produits, et l'implication des maris dans la décision du couple de pratiquer la PF.

"Durant ces 5 ans, on voit que la PF est beaucoup plus décentralisée, la moitié de la population a senti l'impact de la PF et ce n'était pas le cas avant car il y avait des localités où la PF n'existait pas. Mais durant ces 5 ans tout le monde y a accès » (GD Mbour). 
"C'est important aussi la façon dont on a essayé de mener des campagnes de sensibilisation à la télé sur la planification familiale. S'ily avait 100 qui décédaient à cause de cela, maintenant il n'y en a que 10 qui décèdent. C'est très important »(GD Ndayane).

Pour avoir de meilleurs effets, les femmes suggèrent de renforcer la communication et d'éviter les ruptures de stocks des produits.

\section{PERENNISATION DE L'APPROCHE 3D}

De l'avis de nombreux acteurs PF, la pérennisation de l'approche 3D passe par une bonne sensibilisation pour une meilleure compréhension afin de dépasser le niveau de concept. Elle passe aussi par une plus grande implication et appropriation par les districts, les communautés, les autres secteurs d'intervention, et surtout les collectivités locales et l'Etat. Tous doivent pleinement jouer leur rôle, en mettant en place des stratégies de pérennisation, notamment pour la motivation des acteurs communautaires et la sécurisation des produits, car l'essentiel des besoins est actuellement couvert par les partenaires.

"Ily a encore beaucoup de choses qui sont laissées aux mains des bailleurs ou des partenaires d'exécution. Il faudrait vraiment que dans la vision du Ministère de la Santé et de la DSR qu'on puisse tirer les leçons et qu'on puisse voir comment on va pérenniser cela parce que les bailleurs et les partenaires d'exécution ne seront pas toujours là. Et que l'idée c'est quand même quand quelque chose marche qu'on puisse continuer cela mais avec nos propres moyens. Donc ça, ça doit être très, très, très murement réfléchi 》 (Représentant ONG).

"C'est sécuriser les produits qu'il n'y ait pas de ruptures et qu'au départ des ONG que ça continue. Que l'Etat trouve les moyens de pérenniser. Beaucoup d'ONG sont passées. Il y a tellement d'ONG qui sont passées au Sénégal que si elles partent c'est le retour à la case de départ. Il faut sensibiliser les gens. Il faut leur dire que c'est pour vous pas pour eux et que petit à petit l'Etat accompagne les gens à être autonomes » (Représentant ONG).

Les femmes bénéficiaires pensent, pour la plupart, que la pérennisation de l'approche 3D est assujettie à une meilleure sensibilisation, surtout à l'endroit des hommes, détenteurs du pouvoir de décision et des belles-mères, à une disponibilité accrue des produits, et à une formation continue des acteurs communautaires.

"C'est aux hommes qu'on doit parler pour qu'ils soutiennent les femmes... Les belles-mères, je pense que c'est important aussi d'entrer dans les maisons et de les sensibiliser comme on le fait avec les hommes. Elles nous jettent souvent des pierres. Elles ne nous aident pas. Elles ne sont pas d'accord parce qu'elles disent qu'en leur temps, elles ne faisaient pas la planification familiale 》(GD Ndayane). 


\section{Discussion}

Les résultats de cette étude montrent que l'approche 3D est peu connue par les parties prenantes de la PF en tant que concept, même si elles la pratique en réalité régulièrement sur le plan opérationnel. Sa mise en place s'inscrit dans un contexte marqué par une volonté des plus hautes autorités de booster la PF et de réduire les BNS. Les changements opérés sur les plans institutionnel, politique et organisationnel augurent que l'approche présente des promesses fortes pour l'atteinte des objectifs de développement du Sénégal. Toutefois sa reconduction dans le prochain plan d'action PF exige la prise en compte de certains défis pour chacun des « $»:$

\section{En ce qui concerne la démocratisation :}

- La situation économique difficile de la majorité des populations, surtout rurales, a conduit à mettre en place des initiatives de gratuité des services. Cependant, les structures de santé ont des exigences de ressources financières et se trouvent dans l'obligation de recouvrer les coûts auprès des clientes. Cela conduit à des blocages à la démocratisation.

- Les dispositions juridiques et règlementaires constituant des barrières à l'implication du secteur privé, notamment les pharmacies et cabinets privés, devraient être révisées afin d'ouvrir des opportunités d'élargissement de l'offre. Cependant, pour garantir la stabilité du marché et l'équité d'accès, il faudra aborder la question de la subvention ou non des produits dans le secteur privé à but lucratif.

- Avoir posé le débat sur la PF sur la place publique a certes facilité la discussion au sein des couples et dans la communauté, mais des zones d'ombre demeurent. Par exemple, le PANPF cible les femmes en union, et bien qu'il existe un plan stratégique de SRAJ, la PF des jeunes célibataires, n'y est pas explicitement prise en compte. Pour des raisons religieuses et culturelles, la précocité de l'activité sexuelle est un sujet délicat. Cependant, il est urgent de mettre en place des interventions spécifiques pour tous les jeunes, qui représentent une part importante de la population.

- Les nombreux cas de grossesses non désirées, d'avortements illégaux aux conséquences physiques et mentales dramatiques, d'abandons d'enfants ou d'infanticides sont autant d'indications de BNS. Les intervenants dans le domaine de la PF sont interpellés quant aux mesures à mettre en œuvre pour assurer l'accès et le maintien volontaire des utilisatrices de PF dans le programme.

- La contribution de la PF au développement économique et social n'est plus à démontrer. De même, l'importance de l'implication effective d'autres structures gouvernementales (notamment des Ministères de l'Education; de la Femme, de la Famille et de l'Enfance; de la Jeunesse ; de l'Economie et des Finances; et de la Communication) pour la promotion de la démocratisation n'est plus à discuter. Aussi, le débat devrait plutôt s'orienter vers la recherche de solutions pour l'intégration de l'apport de chaque secteur afin que la multisectoralité ne soit pas un simple slogan, mais plutôt une réalité.

\section{Par rapport à la démédicalisation :}

- L'intégration de la case de santé dans la pyramide sanitaire apparait comme une priorité dans un contexte où les questions de motivation du personnel communautaire se posent avec acuité.

- De plus, même si des progrès ont été faits dans l'utilisation des agents de santé communautaire pour l'offre de services, l'étude a montré que leur crédibilité n'est assurée ni auprès des acteurs de la PF, ni auprès des femmes bénéficiaires. Les efforts doivent s'intensifier pour assurer la qualité des services fournis à ce niveau pour ne pas donner le flanc aux détracteurs et remettre en cause les acquis. Cela va de la formation au suivi/supervision et jusqu’à leur motivation. 
- Les cadres légal et réglementaire doivent être renforcés pour permettre une implication encadrée des agents de santé communautaire.

\section{Concernant la décentralisation :}

- L'insuffisance des infrastructures sanitaires et du personnel qualifié exigent la mise en place d'alternatives comme les cases de santé améliorées. Cela pourrait permettre de faire avancer la décentralisation dans une situation où les pesanteurs socioculturelles sont encore bien réelles.

- Pour la durabilité du programme, l'insuffisance de la part contributive du Sénégal mérite une attention particulière pour éviter que le financement de la PF continue à être porté par les PTF. En effet, il semble difficile de parler de pérennisation lorsque la disponibilité des produits est liée aux financements extérieurs. Au-delà de la volonté politique affichée et de l'existence d'une ligne budgétaire dédiée, le renforcement conséquent et effectif des ressources financières contribuerait à une prise en charge plus importante de la partie nationale dans la disponibilité des produits contraceptifs. Le plaidoyer, qui commence à montrer des résultats, pour l'augmentation du financement de la PF par l'Etat et les collectivités locales, doit être soutenu. 


\section{Bonnes pratiques à explorer}

Cette étude met en exergue l'utilisation de plusieurs bonnes pratiques, dont certaines sont reconnues comme des Pratiques à Haut Impact (PHI).

\section{Concernant la Démocratisation de la PF :}

- Les stratégies avancées (équipes mobiles et d'autres modèles décrits dans la Revue documentaire) pour atteindre les populations les plus éloignées.

- L'implication des hommes et des leaders religieux, qui représentent des audiences déterminantes dans les actions de communication. Certains sont des champions de la PF. Des résultats intéressants sont observés par rapport à leur rôle dans l'adhésion des populations à la PF.

- La création par l'Etat d'un environnement politique et juridique favorable, ainsi que son engagement financier.

\section{Concernant la Démédicalisation :}

- L’utilisation des agents de santé communautaire dans l'offre d'une gamme élargie de méthodes, ce qui facilite l'accès des femmes rurales et des zones reculées à la PF.

\section{Concernant la Décentralisation :}

- Le développement d'un modèle efficace d'approvisionnement avec l'IPM qui a conduit à une diminution considérable des ruptures de stock.

- Le plaidoyer pour l'augmentation du financement des produits et services de PF par l'Etat et les collectivités locales.

- Le fait, dans certaines régions de doubler les postes de santé en sages-femmes

- La mise en place des cases de santé améliorées. 


\section{Recommandations}

Les recommandations sont classées par audience :

\section{ETAT/MSAS}

- Créer d'avantage de structures sanitaires, tous les $5 \mathrm{~km}$, si possible moins, pour mieux rapprocher les services des populations.

- Améliorer la disponibilité et la répartition du personnel de santé qualifié.

- Assurer la formation aussi bien du personnel qualifié que du personnel communautaire.

- Rendre la supervision systématique et régulière à tous les niveaux

- Augmenter la ligne budgétaire dédiée à la PF et engager effectivement les fonds le plus rapidement possible.

- Documenter et réviser les cadres juridiques et réglementaires qui entravent l'offre de services par le secteur privé et les agents de santé communautaire.

- Mettre en place des mécanismes visant à renforcer la motivation du personnel communautaire.

- Mettre en place des mesures pour le respect de la circulaire sur les prix.

- Assurer que les PNP sont disponibles et en adéquation avec ce qui se passe sur le terrain.

- Assurer le leadership pour la mise en œuvre effective de la multisectoralité dans l'offre de services de $\mathrm{SR} / \mathrm{PF}$.

- Assurer le leadership pour inventorier et documenter, y compris la composante coût, des bonnes pratiques résultant des interventions mises en œuvre. En partager largement les résultats pour en permettre l'utilisation dans la mise en œuvre des programmes et projets, au Sénégal et ailleurs dans le monde.

- Faciliter le passage à l'échelle des interventions reconnues comme de bonnes pratiques.

- Vulgariser l'approche 3D en vue d'une meilleure appropriation et application.

- Spécifier clairement comment l'approche 3D s’articule dans le prochain PANPF.

\section{ACTEURS PF/PARTENAIRES TECHNIQUES}

- S’approprier davantage l'approche 3D et la mettre en relation dans les interventions menées.

- Renforcer l'appui à la formation aussi bien du personnel qualifié que du personnel communautaire.

- Renforcer le plaidoyer pour l'augmentation du financement de l'Etat et des collectivités locales pour la PF.

- Renforcer l'implication des communautés, notamment les hommes et leaders religieux dans le cadre des actions de communication.

- Evaluer et documenter de façon minutieuse les interventions, réussies ou moins réussies, compris la composante coût, afin d'en tirer les leçons et de les partager avec les autres acteurs pour le bénéfice de tous.

- Evaluer et documenter les bonnes pratiques, y compris la composante coût, afin d'en permettre, le cas échéant, la réplication, la mise à l'échelle et le partage.

- Collaborer pour le passage à l'échelle des bonnes pratiques.

- Partenaires financiers 


\section{PARTENAIRES FINANCIERS}

- Assurer la disponibilité des produits de la gamme des méthodes disponibles au Sénégal.

- Soutenir la mise en œuvre de programmes de recherche et d'interventions dans le domaine de la SR/PF contribuant à la réalisation des objectifs de développement du Sénégal et globaux, tels que les ODD. 


\section{Limites de l'étude}

Les principales limites de cette étude sont : l'absence de réponses aux sollicitations de rendez-vous par certains acteurs PF dont le point de vue n'a pu être pris en compte ; la difficulté à localiser et à se procurer de la documentation sur l'approche $3 \mathrm{D}$ et les interventions y référant. Tout ceci concourt à la non-exhaustivité de la description des acteurs et des interventions relatives aux 3D. Cette étude ne visait pas à déterminer si l'approche a atteint des objectifs qui lui auraient été fixée à l'avance, de tels objectifs n'existant pas, mais plutôt de documenter, à posteriori, ce qui a été fait. Toutefois, du fait que l'étude s'est limitée aux régions de Dakar et Thiès, il est possible que des spécificités d'autres régions ne soient pas ressorties ici.

\section{Conclusion}

Lancé officiellement en 2012, le PANPF 2012-2015 a permis au Sénégal de réaliser des progrès importants dans le domaine de la PF. L'approche 3D sur laquelle repose ce plan, bien que peu connue conceptuellement des parties prenantes, a été reconnue familière, après explications. Elle touche autant au volet politique qu'au volet opérationnel.

La démocratisation, en consacrant l'implication de différents secteurs ministériels et d'autres acteurs tels que les parlementaires, la société civile, le secteur privé, les élus locaux, les leaders religieux et communautaires, a été jugée essentielle. Elle a permis, entre autres, d'améliorer les connaissances et perceptions des populations sur la PF, d'harmoniser les prix des contraceptifs, d'élargir la prestation de services et d'accroitre le leadership des femmes.

La démédicalisation, grâce à la délégation des tâches et à la distribution à base communautaire, a permis d'élargir les compétences des agents de santé communautaire et de rapprocher les services des populations.

La décentralisation, en favorisant l'appropriation et la responsabilisation à tous les niveaux, a permis l'accès à une gamme plus large de méthodes modernes de PF, notamment par : le passage à l'échelle de l'OIP et de l'OII ; la densification des PPS, y compris par la réhabilitation des cases de santé ; et l'introduction des MLDA au niveau des postes de santé. La décentralisation a aussi contribué à garantir la disponibilité des produits jusqu'aux niveaux les plus périphériques.

Ainsi, les réalisations liées aux 3D ont contribué à la nette amélioration des indicateurs. Les données des EDSC et les résultats de la revue à mi-parcours du PANPF, en février 2015, montrent des bonds successifs du TPC pendant les 3 dernières années, le faisant passer de 12 pour cent en 2012 à 20 pour cent. Les efforts doivent être poursuivis pour atteindre l'objectif de 27 pour cent en 2015.

Enfin, la pérennisation de l'approche 3D passe nécessairement par sa meilleure connaissance et appropriation par les parties prenantes, une réelle application de la multisectoralité, une révision des cadres juridique et réglementaire, et une implication de l'Etat et des collectivités locales dans le financement de la PF. La prise en compte de l'approche et sa clarification dès l'élaboration du prochain PANPF s'avèrent indispensables pour booster encore plus rapidement la PF pour améliorer la santé des populations et réduire la mortalité maternelle, néonatale et infantile. 


\section{Références bibliographiques}

Agence Nationale de la Statistique et de la Démographie (ANSD) [Sénégal], et ICF International. 2012. Enquête Démographique et de Santé à Indicateurs Multiples au Sénégal (EDS-MICS) 2010-2011. Calverton, Maryland, USA: ANSD et ICF International.

Agence Nationale de la Statistique et de la Démographie (ANSD) [Sénégal], et ICF International. 2013. Enquête Démographique et de Santé Continue (EDS-Continue 2012-2013). Calverton, Maryland, USA: ANSD et ICF International.

Agence Nationale de la Statistique et de la Démographie (ANSD) [Sénégal], et ICF International. 2015. Sénégal : Enquête Démographique et de Santé Continue (EDS-Continue 2014). Rockville, Maryland, USA : ANSD et ICF International.

Cabinet Sen Ingénierie Consult. Décembre 2014. Evaluation à mi-parcours de la campagne de communication sur la Planification Familiale 2013-2014. Rapport final. Dakar : Ministère de la Santé et de l'Action Sociale.

Cellule de Santé Communautaire et Ministère de la Santé et de l'Action Sociale. 2014. Plan National Stratégique Santé communautaire 2014-2018. République du Sénégal.

Cellule de Santé Communautaire et Ministère de la Santé et de l'Action Sociale. 2015. Document cadre de la stratégie des sages-femmes itinérantes. Dakar: Ministère de la Santé et de l’Action Sociale.

Daff BM, Seck C, Belkhayat H, Sutton P. 2014. Le système de distribution en «Push » des contraceptifs mis en place au Sénégal réduit les ruptures de stock et améliore la qualité des services de planning familial. Global Health Sci Pact. 2014; 2(2):245-252. Disponible sur http://dx.doi.org/10.9745/GHSP-D-13$\underline{00171}$

Direction de la Santé de la Reproduction et de la Survie de l'Enfant. 2012. Rapport de la revue du Programme Bajenu Gox. Dakar : DSRSE.

Direction de la Santé de la Reproduction et de la Survie de l'Enfant (DSRSE) et Ministère de la Santé et de l'Action Sociale (MSAS). 2013. Plan opérationnel de plaidoyer sur la planification familiale 2013-2015. Dakar : DSRSE.

Division de la Planification Familiale, Direction de la Santé de la Reproduction et de la Survie de L'Enfant et Ministère de la santé et de l'action sociale. 2013. Plan d'introduction et d'évaluation de Sayana ${ }^{\circledR}$ Press au Sénégal. Dakar : DSRSE.

Division de la Santé de la Reproduction (DSR) et Ministère de la Santé et de l'Action Sociale (MSAS). 2011. Plan Stratégique de la Santé de la Reproduction du Sénégal 2012-2015. Dakar : MSAS.

Division de la Santé de la Reproduction (DSR) et Ministère de la Santé et de l'Action Sociale (MSAS). 2012. Plan d'Action National de Planification Familiale 2012-2015. République du Sénégal. Dakar : MSAS.

Division de la Santé de la Reproduction des Adolescent(e)s/Jeunes, Direction de la Santé de la Reproduction et de la Survie de L'Enfant (DSRSE) et Ministère de la santé et de l'action sociale et (MSAS). 2014. Plan Stratégique de Santé sexuelle et de Reproduction des Adolescent(s)/Jeunes au Sénégal 2014-2018. Dakar : MSAS.

Dubent L, Mbow FB, Diop N. 2015. “Evaluation de la mise en œuvre et des réalisations de l'Approche 3D au sein du Plan d'Action National de Planification Familiale (PANPF) au Sénégal - Revue documentaire”. 
Gouvernement du Sénégal. 2005. Loi no 2005-18 du 5 août 2005 relative à la santé de la reproduction. Dakar: Gouvernement du Sénégal.

High-Impact Practices in Family Planning (HIP). 2014. Financing commodities and services: mobilizing resources to sustain current and future family planning demand. Washington, DC: USAID. Disponible sur: https://www.fphighimpactpractices.org/sites/fphips/files/hip_financing_fre.pdf (Consulté le 28/10/15)

Mané B, Diop N, Termini N, Ramarao S, Clark H. 2012. Anneau vaginal à la Progestérone. Cartographie pays : Sénégal. Dakar : Population Council.

Mbow FB, Ningue EAB, Diop N, Mané B, Ngouana R. 2015a. "La délégation des tâches dans le domaine de la planification familiale au niveau communautaire dans les pays du Partenariat de Ouagadougou : Expériences et leçons apprises pour une mise en œuvre effective - Présentation par pays". Dakar: Population Council.

Mbow FB, Ningue EAB, Diop N, Mané B, Ngouana R. 2015b. "La délégation des tâches dans le domaine de la planification familiale au niveau communautaire dans les pays du Partenariat de Ouagadougou : Expériences et leçons apprises pour une mise en œuvre effective -Rapport de synthèse". Dakar: Population Council.

Ministère de la Santé et de l'Action Sociale (MSAS). 2014. Lettre circulaire N008938/MSAS/DGS/CSC du 11 aout 2014 portant extension de l'offre initiale de contraceptifs injectables intramusculaires et souscutanés au niveau communautaire.

Ministère de la Santé et de la Prévention. 2010. Lettre circulaire Nº07607/MSP/DSR du 26 juillet 2010 fixant les prix de cession des produits contraceptifs.

Ndiaye S, et Ayad M. 2006. Enquête Démographique et de Santé au Sénégal 2005. Calverton, Maryland, USA: Centre de Recherche pour le Développement Humain [Sénégal] et ORC Macro.

Partenariat de Ouagadougou. 2011. La planification familiale : l'A frique de l'ouest francophone en mouvement. Un appel à l'action.

PATH. Janvier 2015. Recherche sur l'auto-injection de Sayana ${ }^{\circledR}$ Press. Seattle : PATH.

Pharmacie Nationale d'Approvisionnement (PNA). Décembre 2014. Modèle de Distribution Concertée (MoDisc) « jegesi naa ». Note Conceptuelle. Dakar : République du Sénégal.

Projet Initiative Sénégalaise de Santé Urbaine (ISSU). 2014a. Rapport de la capitalisation des approches du « paquet porteur » des interventions du projet ISSU. Dakar : IntraHealth International.

Réseau Siggil Jigeen. 2015a. «Le gain rapide. Cas de la commune de Pikine Djidah Thiaroye Kao ». Dakar.

Réseau Siggil Jigeen. 2015b. Plaidoyer de budget avec le maire de la commune de Mbao. Dakar.

Réseau Siggil Jigeen. 2015c. Rapport trimestriel d'Advance Family Planning- Février Mars Avril 2015. Dakar.

Sidze EM, Lardoux S, Speizer IS, Faye CM, Mutua MM, Badji F. 2015. Accès et recours des jeunes femmes à la contraception: rôle des restrictions imposées par les prestataires en milieu urbain au Sénégal. Perspectives Internationales sur la Santé Sexuelle et Génésique. Numéro spécial de 2015, pp. 20-28. Doi: $10.1363 /$ FR02015.

The RESPOND Project. 2014. Reality Check experiences: Use of a program planning and advocacy tool for family planning initiatives. RESPOND Project Brief No. 22. July. New York: EngenderHealth (The RESPOND Project). 
USAID-Sénégal. 2011. Expérience acquise de Programme de Santé Communautaire ChildFund/USAIDSénégal. Évaluation Finale - Programme de Santé Communautaire du Sénégal/ChildFund 2006-2011. Dakar : USAID Sénégal.

\section{Sites web consultés}

Bureau de l'OMS au Sénégal. Bulletin d'information du Bureau de l'OMS au Sénégal no108, aout 2013.

Disponible sur http://www.afro.who.int/fr/senegal/press-materials/item/5842acc $\% \mathrm{C} 3 \% \mathrm{~A} 91 \% \mathrm{C} 3 \% \mathrm{~A} 9$ ration-de-la-r $\% \mathrm{C} 3 \% \mathrm{~A} 9$ alisation-des-omd-relatifs- $\% \mathrm{C} 3 \% \mathrm{~A} 0-\mathrm{la}-$ r\%C3\%A9duction-de-la-mortalit\%C3\%A9-maternelle-et-n\%C3\%A9onatale.html. Consulté le $14 / 10 / 2015$.

Family Planning High Impact Practices. Pratiques à Haut Impact dans la Planification Familiale. 2014. Disponible sur https://www.fphighimpactpractices.org/sites/fphips/files/hip_list_fre.pdf. Consulté le $23 / 10 / 2015$.

Partners in Population and Development Africa Regional Office. Le dividende démographique et le développement. Disponible sur http://www.ppdafrica.org/docs/policy/demo-f.pdf. Consulté le $22 / 10 / 2015$.

\section{Présentations}

Daff Bocar Mamadou. Planification familiale au Sénégal. Amélioration de l'accès par les 3D. Conférence "Population, développement et planification familiale en Afrique de l'ouest francophone : l'urgence d'agir", Février 2011, Ouagadougou.

Marie Stopes International. La Franchise Sociale MSI au Sénégal, Février 2015.

Marie Stopes international. Les équipes mobiles pour atteindre les populations défavorisées, Saint Louis, Avril 2015.

Marie Stopes International. Mobiliser les Jeunes pour un comportement sain et responsable, Mai 2014.

Projet Initiative Sénégalaise de Santé Urbaine (ISSU). 2014 b. "Synthèse des résultats de l'enquête à miparcours ». DSRSE, Atelier de restitution des résultats de l'enquête à mi-parcours, de l'évaluation de l'ISBC et du processus de capitalisation du « Paquet Porteur » du projet ISSU, Dakar, 24 juin 2014. 



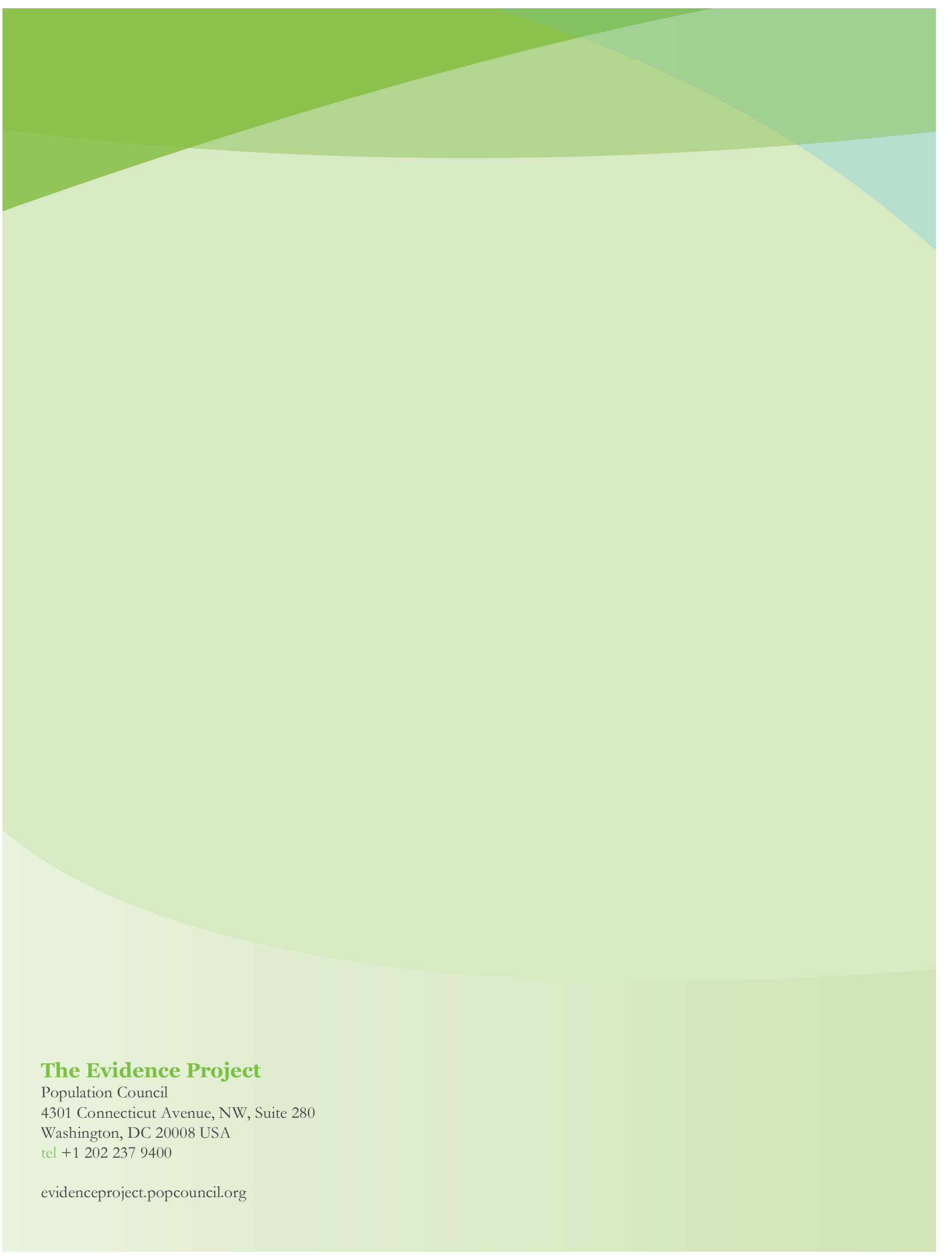

